\title{
Annotated checklist of the millipede family Chelodesmidae Cook, 1895 from São Paulo state, Brazil (Diplopoda: Polydesmida)
}

\author{
Rodrigo Salvador Bouzan ${ }^{1,3}$; Luiz Felipe Moretti Iniesta ${ }^{1,2,4}$; João Paulo Peixoto Pena-Barbosa ${ }^{1,5}$ \& Antonio Domingos Brescovit ${ }^{1,6}$ \\ 1 Instituto Butantan, Laboratório Especial de Coleções Zoológicas (LECZ). São Paulo, SP. Brasil. \\ 2 Universidade de São Paulo (USP), Instituto de Biociências (IB-USP). São Paulo, SP. Brasil. \\ 3 E-mail: rodrigobouzan@outlook.com \\ ${ }^{4}$ ORCID:0000-0002-0529-4162. E-mail: luiz-moretti@hotmail.com \\ ${ }^{5}$ E-mail: ipppbarbosa@gmail.com \\ ${ }^{6}$ ORCID: 0000-0002-1511-5324. E-mail: adbresc@terra.com.br
}

\begin{abstract}
A checklist of the family Chelodesmidae Cook, 1895 (order Polydesmida) from state of São Paulo, Brazil has been performed based on literature and examined material from the collection of the Instituto Butantan, São Paulo (IBSP). A total of 15 genera ( 7 tribes and 5 genera considered incertae sedis) with 64 species are listed. Among these species, 30 presented a single one record in the state, 19 with more than one record and four recorded for the first time for the state of São Paulo, and 11 species occurring in other Brazilian states. The most distributed species is Brasilodesmus paulistus paulistus (Brölemann, 1902) with 52 records of occurrence. In addition, a complete bibliography list of the chelodesmidan fauna from the state is compiled, as well as distribution maps for all species are provided.
\end{abstract}

Key-Words. Neotropical; Chelodesminae; Diversity; Atlantic forest; Cerrado.

\section{INTRODUCTION}

Among the representatives of the class Diplopoda, Chelodesmidae Cook, 1895 is one of the richest families, with almost 800 described species in the order Polydesmida Pocock, 1887 (Hoffman, 1980; Shear, 2011; Pena-Barbosa et al., 2013). Members of the family has a wide range of colors, paranota (or keels) with a variety of shapes, and males with numerous and differentiable processes on the gonopods (Schubart, 1955; Hoffman, 1969, 1976; Pena-Barbosa et al., 2013; Bouzan et al., 2017).

Hoffman (1980) proposed the division of Chelodesmidae into two subfamilies, Chelodesminae Cook, 1895 for the Neotropical species, and Prepodesminae Cook, 1896 for the members from West Africa. During his lifetime, Hoffman proposed 19 tribes for Chelodesminae and only one for Prepodesminae. Among the 800 species, only 345 were assigned to tribes, in while remaining species still require new taxonomic reviews for an exact tribal designation.

Few information is known regarding the millipede fauna in general and their distribution in Brazilian territory at the moment, mainly in function of a scarcity of specialists and, consequently, of taxonomic studies about the group. In this country, the portion southeastern is one of the best-explored region in relation to the Brazilian diversity of Chelodesmidae. For this, great part of the information concerning the millipede fauna is resulted of extensive studies made by Schubart in the last century (Knysak \& Martins, 1999). The state of São Paulo, where Schubart settled after his coming to the country, performed some efforts to the knowledge of faunistic composition (Schubart 1955, 1960; Lindner et al., 2012). Before him, only Brölemann (1902a) and Attems (1931) gave attention to the fauna of the state with some species described, indeed.

Thus, to promote further studies regarding Chelodesmidae from state of São Paulo, the aim of this study is to present a current list of species for the family, according to the distribution, with inclusion of new records, and richness of species in the state, as well as a relevant bibliography list of the fauna from the state is compiled.

\section{MATERIAL AND METHODS}

The list of species of the Chelodesmidae from the state of São Paulo (Fig. 1) was extracted through a series of queries on associated literature. The material examined was obtained exclusively from the millipede deposited in the collection of Instituto Butantan. The list was prepared 


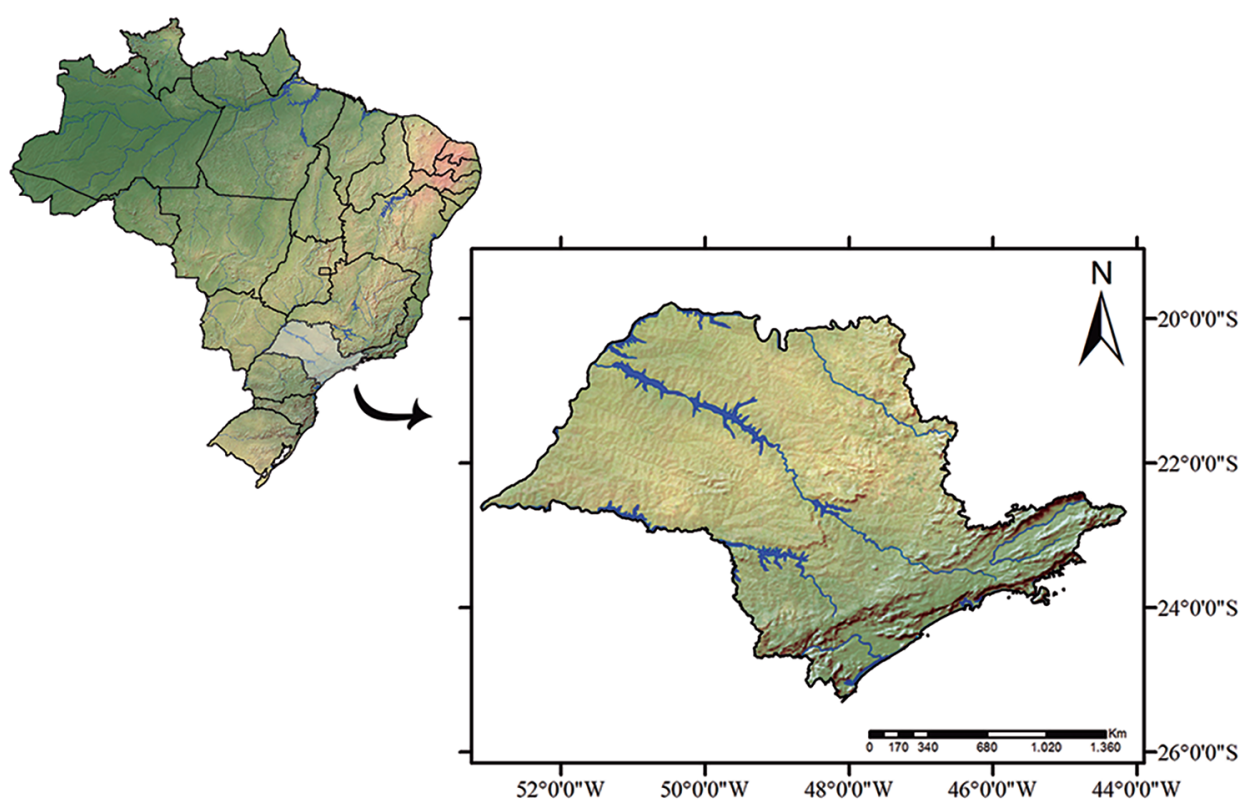

Figure 1. Map of the Brazil with São Paulo state highlighted in grey, and the map of the state detailed.

according to this structure: i) valid species name with author and the year of publication; ii) a complete chronological synonymic list with number of pages and plates/ figures (if known). For this purpose, only taxonomic references were consulted; iii) type locality, types and the repository, if known; iv) records from literature; v) material examined and new records.

The material is deposited in the following institutions (curators in parentheses): FMNH, Field Museum of Natural History, Chicago, EUA (P. Sierwald); IBSP, Instituto Butantan, São Paulo, Brazil (A.D. Brescovit); MZSP, Museu de Zoologia, Universidade de São Paulo, São Paulo, Brazil (R. Pinto da Rocha); NHMW, Naturhistorisches Museum, Wien, Austria; ZMH, Zoologisches Museum Hamburg, Hamburg, Germany (M. Glaubrecht); ZSM, Zoologische Staatssammlung München, Germany (R. Melzer \& S. Friedrich).

The list of species was organized following the respective tribes and genera. The species with no tribal designation was grouped in a single group incertae sedis. The geographical coordinates were obtained from the original literature (when provided). Species without any information about geographic coordinates were georeferenced using the geoLoc tool of speciesLink (http://splink.cria.org.br/geoloc) for the localities. Species with no exact location provided was not recorded in the maps. The maps were obtained with the ecoregions highlighted, following the indication of colours/nomenclature according to Instituto Life, 2015: i) purple - Rupestrian Grasslands (Campos Rupestres); ii) beige - Brazilian Savanna (Cerrado); iii) light pink - Alto Paraná Atlantic Forest (Floresta Atlântica Alto Paraná); iv) dark mustard - Serra do Mar Coastal Forest (Floresta Costeira da Serra do Mar); v) orange Araucária Moist Forest (Floresta Úmida de Araucária), (Araucaria angustifolia (Bertol.) Kuntze 1898); vi) light mustard - Ilha Grande Mangroves (Mangues da Ilha Grande).
RESULTS

Chelodesmidae Cook, 1895

Chelodesminae Cook, 1895

Tribe Arthrosolaenomeridini Hoffman, 1976

(Fig. 2A)

\section{Angelodesmus Schubart, 1962}

Angelodesmus Schubart, 1962: 258. Type species: Angelodesmus costalimai Schubart, 1962, by original designation and monotypy; Hoffman, 1976: 173, 1980: 151; Shelley et al., 2000: 84.

\section{Angelodesmus planaltensis (Schubart, 1960)}

Arthrosolaenomeris planaltensis Schubart, 1960: 253, Figs. 1-2 (Male holotype, MZSP, from Jaci, Mirassol, 49³1'15.60"W; 2049'02.28"S, São Paulo, Brazil). Angelodesmus planaltensis: Hoffman, 1976: 176.

Historical records: BRAZIL. São Paulo: Mirassol, Jaci (49³1'15.60"W; 2049'02.28"S), 11.i.1950, O. Schubart coll., $10^{\prime \prime}$ (Schubart, 1960); Monte Aprazível, Fazenda São José do Varjão (4942'27.36"W; 2046'01.22"S), 09.i.1953, O. Schubart coll., 391 juv. (Schubart, 1960).

\section{Tribe Chelodesmini Hoffman, 1980} (Figs. 2A-B)

\section{Eurydesmus deSaussure, 1860}

Eurydesmus deSaussure, 1860: 335. Type species: Eurydesmus angulatus deSaussure, 1860 , by monotypy; Humbert \& Saussure, 1872: 55; Attems, 1899: 264; Brölemann, 1902a: 92; Carl, 1903: 544; Brölemann, 1916: 552; Attems, 1931: 53; Attems, 1938: 104; 
Schubart, 1945a: 78; Hoffman, 1950: 186; Schubart, 1951a: 2, 1956b: 456; Hoffman, 1971: 250; Jeekel, 1971: 262; Hoffman, 1980: 151.

Chelodesmus Cook, 1895: 5. Type species: Chelodesmus marxi Cook, 1895, by monotypy and original designation. Hoffman, 1950: 186 (Syn.).

Pseudoeurydesmus Schubart, 1944: 361. Type species: Pseudoeurydesmus baguassuensis Schubart, 1944, by original designation; Schubart, 1953: 113, 1955: 530; Hoffman, 1971: 250 (Syn.).

Pseudoeurydesmella Schubart, 1951a: 12. Type species: Pseudoeurydesmella bella Schubart, 1951a, by monotypy and original designation; Schubart, 1955: 530, 1956b: 456; Hoffman, 1971: 250 (Syn.).

Aneurydesmus (as subgenus of Eurydesmus) Schubart, 1956b: 456. Type species: Eurydesmus ruidus Schubart, 1945a, by original designation. Hoffman, 1971: 250 (Syn.).

\section{Eurydesmus acutatus Schubart, 1951}

Eurydesmus acutatus Schubart, 1951b: 91, Figs. 1-6 (Male holotype, MZSP, from Bairro da Vitória, Amparo, 4646'19.18"W; 2242'28.53"S, São Paulo, Brazil); Hoffman, 1971: 253.

Historical records: BRAZIL. São Paulo: Amparo, Bairro da Vitória (4646'19.18"W; 2242'28.53"S), 19.i.1947, J. Schubart coll., 19; 26.x.1948, O. Schubart coll., 1\%; 28.xii.1949, J. Schubart coll., $20^{7} 39$ (Schubart, 1951b).

\section{Eurydesmus aguirrei (Schubart, 1945)}

Pseudoeurydesmus aguirrei Schubart, 1945b: 308 Figs. 6, 7 (Male holotype, MZSP, from Poços de Caldas, 46³3'41.01"W; 2147'12.35"S, Minas Gerais, Brazil); Schubart, 1955: 530, 1956c: 366.

Eurydesmus aguirrei: Hoffman, 1971: 253.

Historical records: BRAZIL. São Paulo: Monte Alegre do Sul (4640'53.86"W; 2241'23.66"S), x.1942, L. Travassos Filho coll., 10"; 30-31.x.1942, no coll., 10' 19 (Schubart, 1945b); 14-16.iv.1944, no coll., 3 juv. (Schubart, 1945b).

\section{Eurydesmus alcatrazensis (Schubart, 1945)}

Pseudoeurydesmus alcatrazensis Schubart, 1945b: 310, Fig. 8 (Male holotype, MZSP, from Ilha de Alcatrazes, São Sebastião, 4541'27.36"W; 2406'02.34"S, São Paulo, Brazil); Schubart, 1949: 225, Schubart, 1955: 530. Eurydesmus alcatrazensis: Hoffman, 1971: 253.

Historical records: BRAZIL. São Paulo: São Sebastião, Ilha

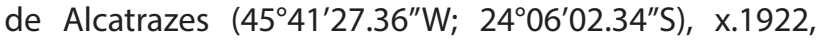
H. Luederwaldt coll., $10^{\prime} ; 16 . i i .1948$, A. Hoge coll., $20^{\prime} 29$ (Schubart, 1945b).

Material examined: BRAZIL. São Paulo: São Sebastião,

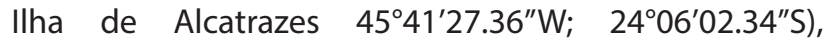
19.ii.1948, Equipe IBSP coll., 19 (IBSP 204).
New records: BRAZIL. São Paulo: Peruíbe, Estação Ecológica Juréia/Itatins (4704'42.79"W; $24^{\circ} 22^{\prime} 53.05^{\prime \prime S}$ ), ii.1993, A. Eterovic coll., $10^{\top 1} 19$ (IBSP 1189).

\section{Eurydesmus baguassuensis (Schubart, 1944)}

Pseudoeurydesmus baguassuensis Schubart, 1944: 362, Figs. 32-34 (Male holotype, MZSP, from Baguassu,

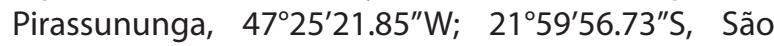
Paulo, Brazil); Schubart, 1955: 530; Jeekel, 1971: 283. Eurydesmus baguassuensis: Hoffman, 1971: 257.

Historical records: BRAZIL. São Paulo: Pirassununga (47²5'21.85"W;2159'56.73"S), 28.x.1940,O.Schubartcoll., 10"; 22-30.xii.1938, O. Schubart coll., 2 juv.; 08-10.i.1939, O. Schubart coll., 191 juv.; 15.xi.1940, O. Schubart coll., 10"; 31.x.1940, O. Schubart coll., 2 juv.; 06.xi.1940, O. Schubart

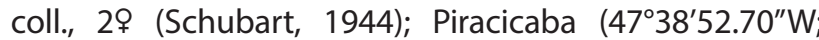
$\left.22^{\circ} 44^{\prime} 05.66^{\prime \prime S}\right), 23 . v .1945$, J. Schubart coll., $30^{\circ} 1$ juv.; Santa

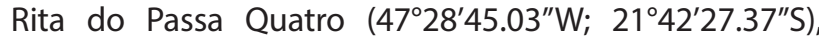
12.xii.1948, no coll., 1 ( (Schubart, 1955).

\section{Eurydesmus brolemanni Hoffman, 1971}

Eurydesmus angulatus: Brölemann, 1902a: 93, Figs. 93-96 (Hoffman, 1971: 258, see Eurydesmus brolemanni specimens recorded from Alto da Serra now Serra de Paranapiacaba, Santo André, 46² 18'59.74"W;
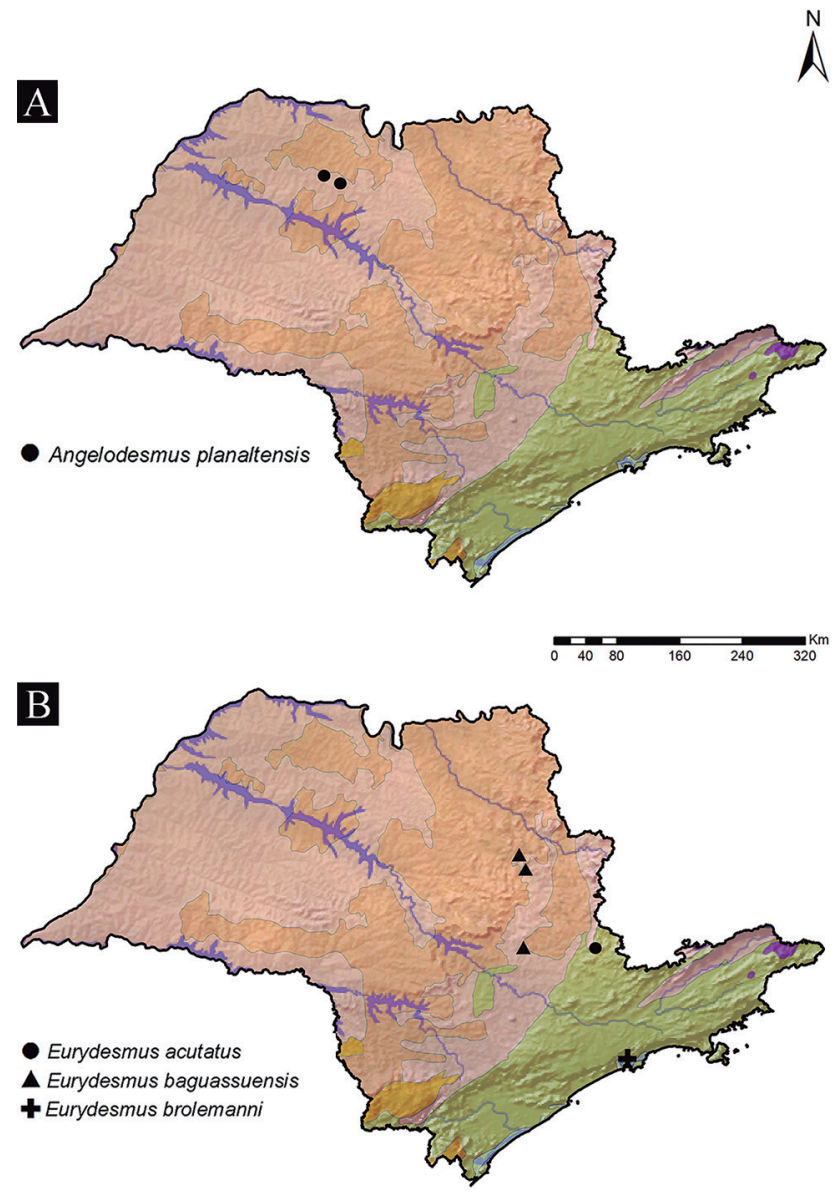

Figure 2. Distribution map of the species belonging to tribes Arthrosolaenomeridini (A) and Chelodesmini (B) from São Paulo state, Brazil. 
2346'59.81"S, São Paulo, Brazil); Schubart, 1945a: 76, Figs. 72-73; Hoffman, 1971: 258.

Eurydesmus brolemanni Hoffman, 1971: 258 (Male holotype, MZSP, from Alto da Serra (now Serra de Paranapiacaba), Santo André, São Paulo, Brazil).

Historical records: BRAZIL. São Paulo: Santo André, Alto da Serra (= Serra de Paranapiacaba) (46¹8'59.74"W; $\left.23^{\circ} 46^{\prime} 59.81^{\prime \prime S}\right)$, no data, no coll., $10^{\prime \prime} 1$ juv. (Brölemann, 1902a).

Remarks: The species Eurydesmus angulatus deSaussure was described based upon a female from an uncertain locality in Brazil (Saussure, 1860), and afterly redescribed by Attems (1898) from females taken at Rio de Janeiro and Santa Catarina states. In 1902, Brölemann determined as E. angulatus a male from São Paulo, through of non-sexual characteristics. Schubart (1945a) reinforced the male of Brölemann as E. angulatus. In the taxonomic revision of the genus, Hoffman (1971) transferred the Brölemann's specimen to a new name E. brolemanni.

\section{Eurydesmus lomatus (Schubart, 1955)}

Pseudoeydesmella lomata Schubart, 1955: 530, Fig. 30 (Male holotype, MZSP, from Serra de Paranapiacaba, 46¹8'59.74"W; 2346'59.81"S, Santo André, São Paulo, Brazil).
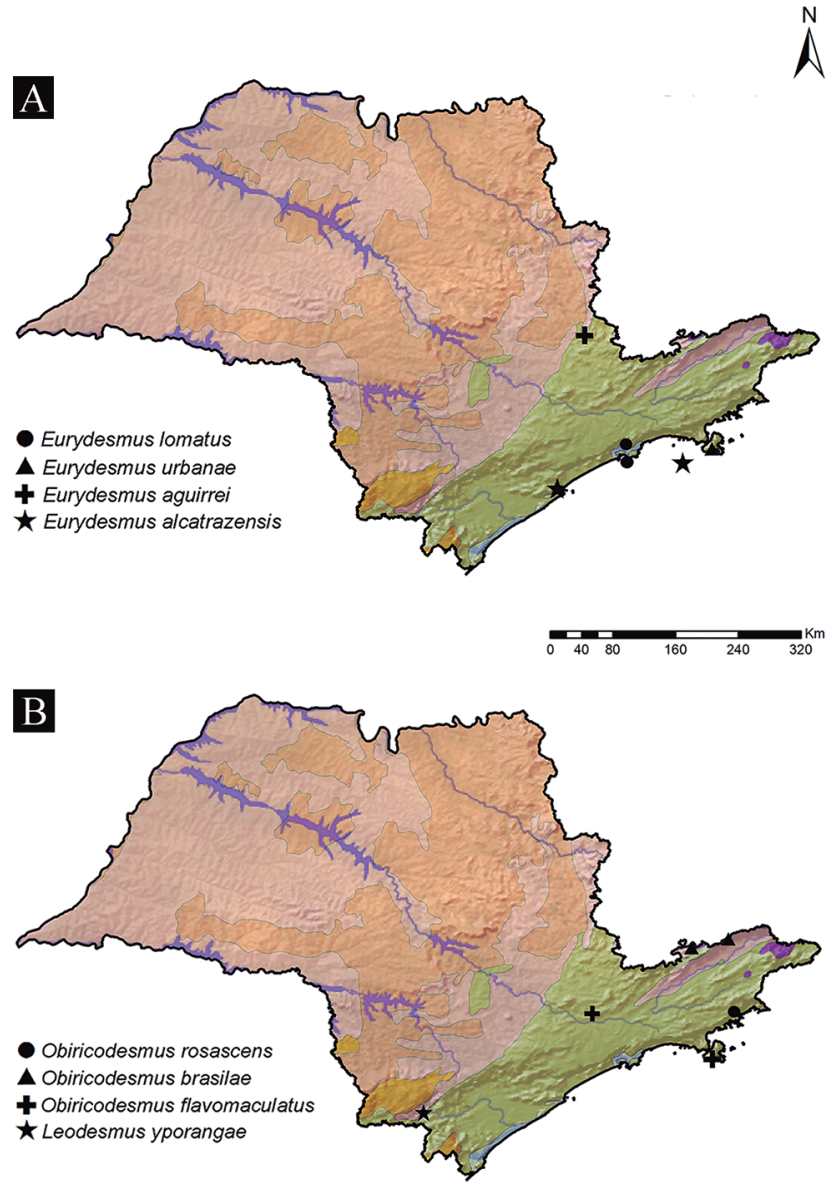

Figure 3. Distribution map of the species belonging to tribes Cornalatini (A) and Leptodesmini (B) from São Paulo state, Brazil.
Eurydesmus lomatus: Hoffman, 1971: 258.

Historical records: BRAZIL. São Paulo: Santo André, Alto da Serra (= Serra de Paranapiacaba) (46¹8'59.74"W; 2346'59.81"S), ii.1925, R. Spix coll., 10'; i.1904, H. Luederwaldt coll., 10'; no data, no coll., 1 ㅇ (Schubart, 1955).

New records: BRAZIL. São Paulo: Guarujá, Praia do Tombo, Ilha da Moela (46¹5'50.15"W; 2402'59.34"S), 17-19.vii.2009, R.P. Indicatti and G.P. Perroni coll., $10^{x} 2$ 우 (IBSP 3252).

\section{Eurydesmus urbanae (Schubart, 1960)}

Pseudoeurydesmus urbanae Schubart, 1960: 463, Fig. 14 (Male holotype, MZSP, from Ilha de São Sebastião (= Ilhabela), 45²0'56.33"W; 2349'51.31"S, São Paulo, Brazil).

Historical records: BRAZIL. São Paulo: Ilhabela (4520'56.33"W; 2349'51.31"S), 25.xi.1949, H. Urban coll., $30^{\top} 1$ 1 (Schubart, 1960).

\section{Tribe Cornalatini Hoffman, 1990}

(Fig. 3A)

\section{Leodesmus Mauriès \& Geoffroy, 2000}

Leodesmus Mauriès \& Geoffroy, 2000: 162. Type species: Alocodesmus yporangae Schubart, 1946, by monotypy; Trajano et al., 2000: 269.

\section{Leodesmus yporangae (Schubart, 1946)}

Alocodesmus yporangae Schubart, 1946: 308, Fig. 1 (Male holotype, MZSP, from cave Areias de Baixo, Iporanga, 4842'01.65"W; 24³5'01.71"S, São Paulo, Brazil); Schubart, 1955: 513; Hoffman, 1969: 281, 1990: 31; Mauriès, 1974: 545, 546; Silhavý, 1974: 897; Pinto-daRocha, 1995: 103.

Leodesmus yporangae: Mauriès \& Geoffroy, 2000: 162; Trajano et al., 2000: 269; ICMBio, 2016: 67.

Historical records: BRAZIL. São Paulo: Iporanga, Parque Estadual Turístico do Alto Ribeira (PETAR), cave Areias de Baixo (4842'01.65"W; 24³5'01.71"S), 1942, C. Pavan coll., 50"7ㅇ (Schubart, 1946); 1942, Dreifuss/Pavan coll., 50" 7\%; cave Areias de Cima (4842'01.65"W; 24³5'01.71"S), 19.ii.1951, O. Schubart coll., 6o' 8\%; 10.xi.1982, E. Trajano coll., 19; 24.viii.1983, N.M. Godoy coll., 80'; cave Água Quente (Ressurgência das Areias), (4840'18.18"W; 243' 45.55"S), 22.x.1983, N.M. Godoy coll., 20' 2o; 29.x.1997, E.H. Santos coll., 20'; cave Alambari de Cima

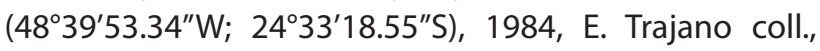
$10^{\prime \prime}$ 19; 09.iv.1990, M.C. Chamani \& J.P.M. Seino coll., $10^{\prime \prime}$ (Mauriès \& Geoffroy, 2000).

Material examined: BRAZIL. São Paulo: Iporanga, Parque Estadual Turístico do Alto Ribeira (PETAR), $\left(48^{\circ} 38^{\prime} 48.09^{\prime \prime} \mathrm{W}\right.$; 
$\left.24^{\circ} 29^{\prime} 10.07^{\prime \prime S}\right)$, undetermined cave, vii.1992, A. Eterovic coll., 10"7? (IBSP 3576).

Remarks: According to the most recent Brazilian Red Book of Threatened Species of Fauna (2016), the species is the only one chelodesmidan considered as critically endangered from Brazil, occurring exclusively in the subterranean system from Vale do Ribeira (Schubart, 1946; Pinto-da-Rocha, 1995).

\section{Obiricodesmus Schubart, 1955}

Obiricodesmus Schubart, 1955: 512. Type species: Rhachidomorpha brasiliae Brölemann, 1902a, by original designation. Schubart, 1955: 512, 1956a: 341; Jeekel, 1971: 275; Hoffman, 1980: 154, 1990: 31; Shelley et al., 2000: 93; Mauriès \& Geoffroy, 2000: 153.

\section{Obiricodesmus brasiliae (Brölemann, 1902)}

Rhachidomorpha brasiliae Brölemann, 1902a: 95, Figs. 105-110 (Male holotype, MZSP, from Piquete,

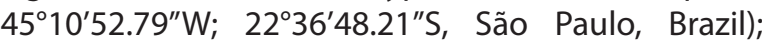
Hoffman, 1969: 281.

Rhachidomorpha bicolor Brölemann, 1902a: 98, Figs. 111-112 (Female holotype, MZSP, from Piquete

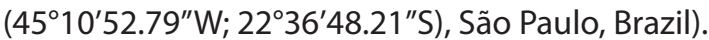

(? Leptodesmus) brasiliae: Brölemann, 1909: 64.

Alocodesmus brasiliae: Attems, 1938: 133, Figs. 155-156.

Obiricodesmus brasiliae: Schubart, 1955: 512, Fig. 1, 1956a: 342, Fig. 1; Hoffman, 1990: 31.

Historical records: BRAZIL. São Paulo: Piquete $\left(45^{\circ} 10^{\prime} 52.79^{\prime \prime} \mathrm{W} ; 22^{\circ} 36^{\prime} 48.21^{\prime \prime} \mathrm{S}\right)$, i.1897, no coll., $10^{\prime \prime}$

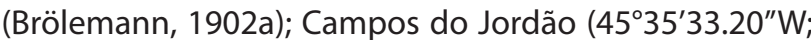
$22^{\circ} 44^{\prime} 34.22^{\prime \prime}$ ), xii.1944, F. Lane coll., $10^{\circ} 5$ 우 (Schubart, 1955).

\section{Obiricodesmus flavomaculatus Schubart, 1956}

Obiricodesmus flavomaculatus Schubart, 1956a: 343, Fig. 3 (Male holotype, MZSP, from Morro do Jaraguá, 4645'54.46"W; 2327'29.67"S, São Paulo, São Paulo, Brazil); Hoffman, 1990: 34.

Historical records: BRAZIL. São Paulo: São Paulo, Morro do Jaraguá 4645'54.46"W; 2327'29.67"S), 04.xii.1949, O. Schubart coll., $10^{\prime \prime}$; São Sebastião $\left(45^{\circ} 24^{\prime} 06.05^{\prime \prime}\right.$; $23^{\circ} 48^{\prime} 22.82^{\prime \prime}$ S), 25.xi.1949, H. Urban coll., 10'; 14.xii.1952, H. Urban coll., 3o' (Schubart, 1956a).

New records: BRAZIL. São Paulo: Ilhabela $\left(45^{\circ} 22^{\prime} 21.24^{\prime \prime} \mathrm{W}_{\text {; }}\right.$ 2349'03.23"S), 09-15.x.2001, Equipe Biota coll., $10^{\prime \prime}$ (IBSP 1934); 1 (क (IBSP 1401); 1 (IBSP 1934).

\section{Obiricodesmus rosascens (Brandt, 1839)}

Polydesmus rosascens Brandt, 1839: 130 (Male holotype, ZMB, from "Brasilia" [= Brazil] without further locality); Brandt, 1841: 130; Gervais, 1847: 113; 1859: 9.
Polydesmus (Rhachidomorpha) rosascens: Peters, 1865: 536; Humbert \& Saussure, 1872: 31, Fig. 158.

Rhachidomorpha rosascens: Attems, 1898: 410, 1938: 472.

Leptodesmus (? Rhachidomorpha) rosascens: Brölemann, 1909: 72

Obiricodesmus rosascens: Hoffman, 1990: 31 Figs. 14-20, 23.

Historical records: BRAZIL. São Paulo: Ubatuba

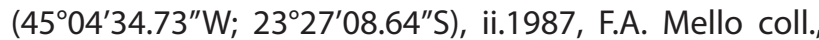
$10^{\top} 1$ 우 (Hoffman, 1990).

\section{Tribe Leptodesmini Attems, 1898 \\ (Figs. 3B, 4A-B, 5A-B, 6A-B, 7A)}

\section{Dialysogon Hoffman, 1979}

Dialysogon Hoffman, 1979a: 229. Type species: Leptodesmus jucundus Brölemann, 1902a by original designation and monotypy; Shelley et al., 2000: 97.

\section{Dialysogon jucundus (Brölemann, 1902)}

Leptodesmus jucundus Brölemann, 1902a: 64, Figs. 41-44 (Male holotype, MZSP, from Santa Rita do Passa Quatro, 47²8'33.56"W; 2142'25.37"S, São Paulo, Brazil); Brölemann 1909: 69; Schubart, 1955: 513.
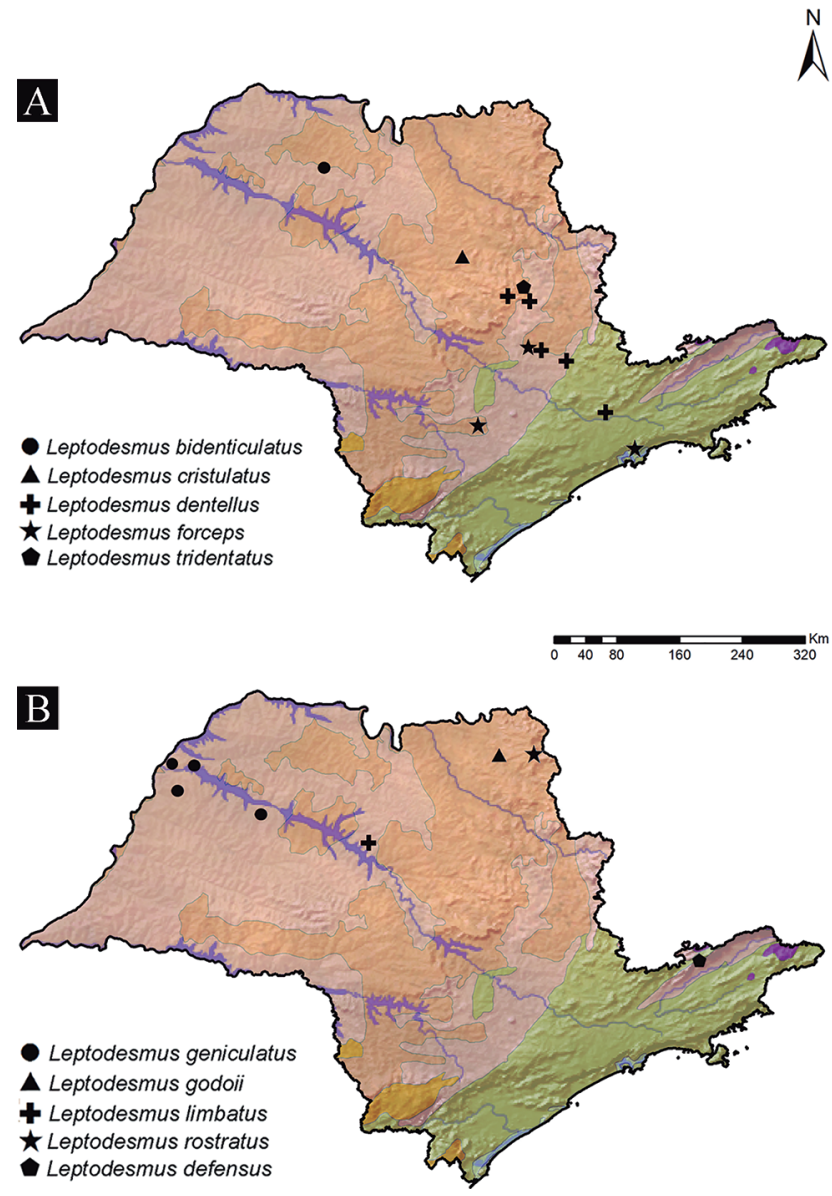

Figure 4. Distribution map of the species belonging to tribe Leptodesmini (A, B) from São Paulo state, Brazil. 
Leptodesmus (Leptodesmus) jucundus: Attems, 1938: 10, Fig. 3; Schubart, 1944: 347.

Dialysogon jucundus: Hoffman, 1979a: 231.

Historical records: BRAZIL. São Paulo: Santa Rita

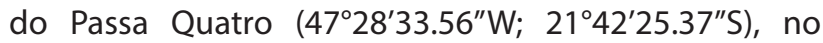
data, no coll., $10^{\top}$ (Brölemann, 1902a); Pirassununga (47²5'31.46"W; 2159'56.92"S), 31.xii.1938, no coll., $10^{\circ}$ 2 juv.; 28.ii.1940, A. Boggi coll., 10'; 06.iii.1940, Aguirre coll., 10'; 29.v.1940, A. Boggi coll., 10;; 28.vi.1940, A. Boggi coll., 19; 23.x.1940, J.B. Moraes coll., 10'; iii.1941, J.B. Moraes coll., 10;; 08.v.1941, J.B. Moraes coll., 10"; 17.v.1941, H. Rosa coll., 20'; 24.ix.1941, H. Rosa coll., $1 \sigma^{*}$; 23.vii.1942, H. Rosa coll., 10"; 30.xii.1938, J. Gaspar coll., $10^{\prime} 3$ juv.; 06-08.i.1939, J. Gaspar coll., 4 juv.; 19.vii.1941, J. Gaspar coll., 19; 04.xii.1941, J. Gaspar coll., 10'; 13.ii.1942, J. Gaspar coll., 10' (Schubart, 1944); 11.iii.1942, J. Gaspar coll., 19; 18.v.1942, J. Gaspar coll., 1o'; 10-11.iv.1942, J. Gaspar coll., 10" 1\%; 25.iv.1942, J. Gaspar coll., 10"; 04.vii.1942, J. Gaspar coll., 10'; 02.viii.1942, J. Gaspar coll., 10; 21-27.xii.1938, J. Gaspar coll., $10^{x} 1$ 1 13 juv.; 22.iv.1942, J. Gaspar coll., 10'; 22.vi.1942, J. Gaspar coll., 10"; Santa Cruz das Palmeiras (47 15'01.90"W; $\left.21^{\circ} 49^{\prime} 33.44^{\prime \prime S}\right)$, 22.xii.1941, no coll., 1 juv.; Descalvado

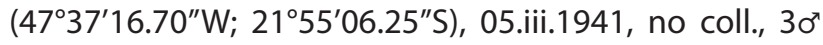
1 juv., (Schubart, 1944); Altinópolis (47²2'26.84"W; $21^{\circ} 01^{\prime} 23.10^{\prime \prime}$ S), 24.vii.1947, no coll., 10'1\%; Santo Antônio da Alegria (4708'53.59"W; $21^{\circ} 05^{\prime} 14.79^{\prime \prime}$ S), 24.vii.1947, no
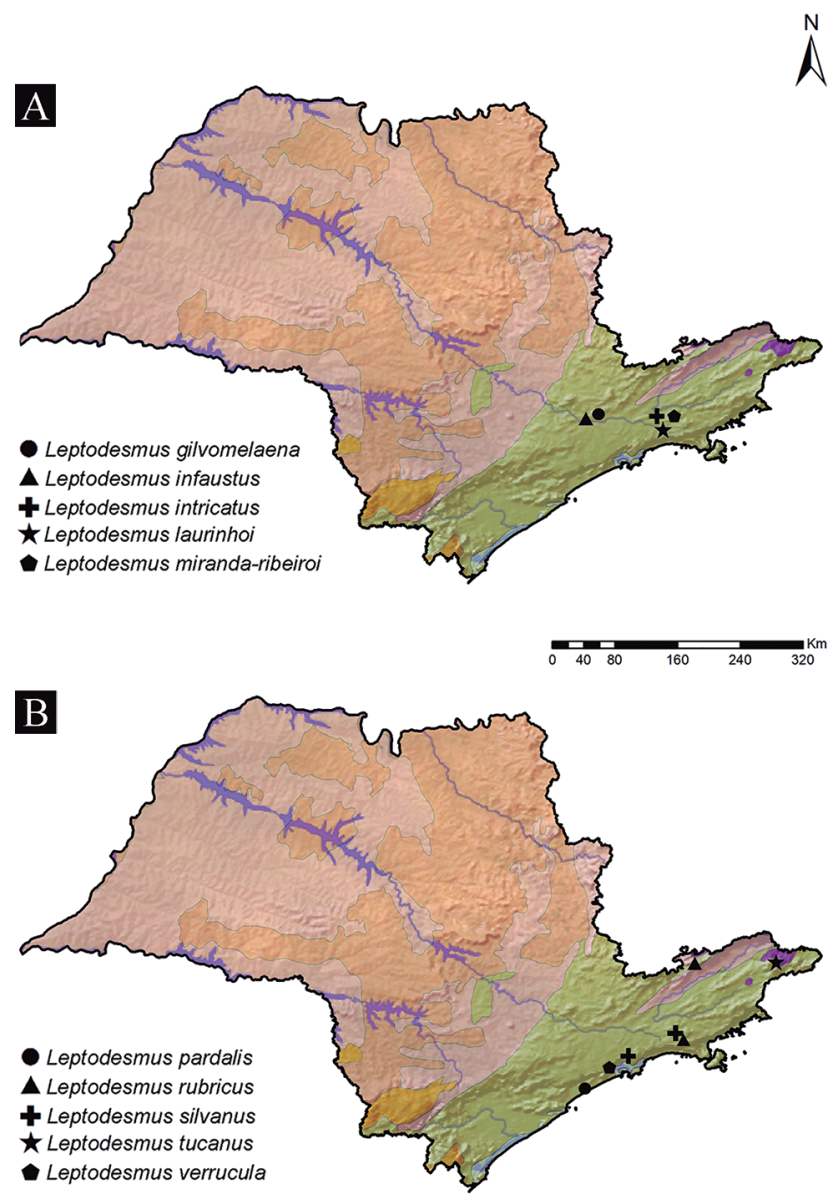

Figure 5. Distribution map of the species belonging to tribe Leptodesmini $(A, B)$ from São Paulo state, Brazil. coll., $90^{\prime} 3$ ㅇ 3 juv.; São Joaquim da Barra (4751'48.54"W; 20³5'02.70"S), 30.i.1944, M.P. de Godoi coll., 1 juv.; Guaíra (48¹8'40.74"W; 20¹9'24.52"S), 06.ix.1944, no coll., 1 ㅇ (Schubart, 1955).

Material examined: BRAZIL. São Paulo: Santa Rita

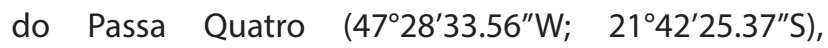
Estação Experimental, 19-24.ii.2002, Equipe Biota coll., $10^{\prime \prime}$ (IBSP 2742); Parque Estadual de Vassununga

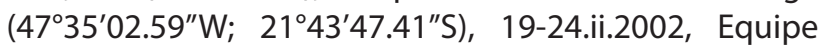
Biota coll., $10^{\prime \prime}$ (IBSP 3277); Pirassununga (47 $25^{\prime} 32.44^{\prime \prime} \mathrm{W}$;

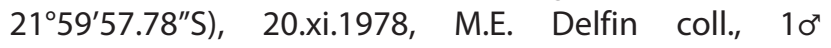
(IBSP 1267).

\section{Leptodesmus deSaussure, 1859}

Leptodesmus deSaussure, 1859: 323. Type species: Polydesmus carneus deSaussure, 1859; proposed as a subgenus of Polydesmus. Type species by subsequent designation of Pocock, 1910: 162; Attems, 1898: 369, 1901: 88; Brölemann, 1902a: 58; Pocock, 1910: 162 (status changed); Brölemann, 1916: 551; Schubart, 1944: 347, 1946: 187, 1953: 99; Kraus, 1954: 21; Hoffman, 1954: 220, 1971: 229, 1980: 152, in tribe Leptodesmini; 2009: 561, 2012: 103.

Goyazodesmus Schubart, 1952: 447. Type species: G. cuspidatus Schubart, 1952: 448, by original designation. Schubart, 1958: 204. Hoffman, 1971: 229 (Syn.).
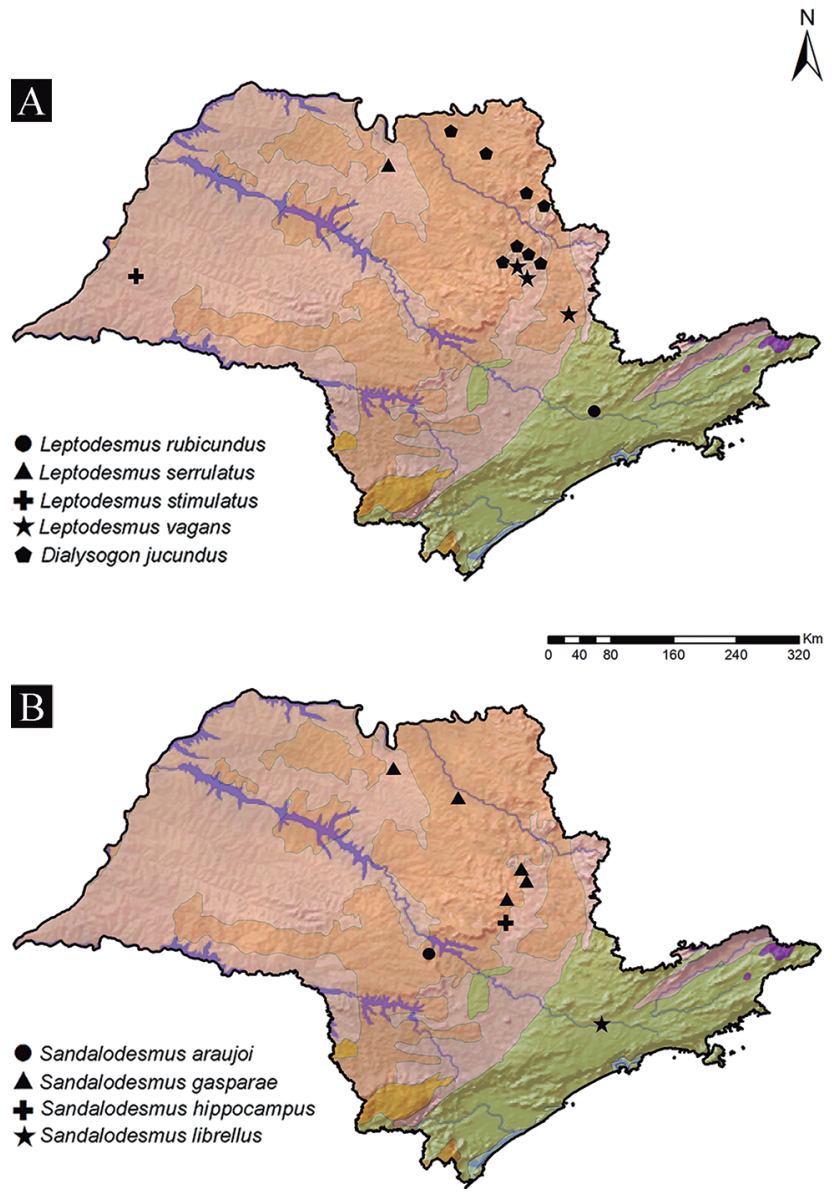

Figure 6. Distribution map of the species belonging to tribe Leptodesmini $(A, B)$ from São Paulo state, Brazil. 


\section{Leptodesmus bidenticulatus Schubart, 1960}

Leptodesmus bidenticulatus Schubart, 1960: 458, Fig. 8 (Male holotype, MZSP, from Fazenda São João do Varjão, Monte Aprazível, 4942'39.06"W; 2046'09.90"S, São Paulo, Brazil); Hoffman, 1971: 237.

Historical records: BRAZIL. São Paulo: Monte Aprazível (4942'39.06"W; 2046'09.90"S), Fazenda São José do Varjão, 09.i.1950, O. Schubart coll., $10^{x} 3911$ juv.; Monte Aprazível (4942'39.06"W; 2046'09.90"S), 10.i.1950, O. Schubart coll., 2 ? (Schubart, 1960).

\section{Leptodesmus cristulatus Schubart, 1955}

Leptodesmus cristulatus Schubart, 1955: 517, Figs. 8-10 (Male holotype, MZSP, from Bueno de Andrade, 48 14'39.58"W; 2140'02.98"S, São Paulo, Brazil); Hoffman, 1971: 243.

Historical records: BRAZIL. São Paulo: Bueno de Andrade (48 $\left.{ }^{\circ} 14^{\prime} 39.58^{\prime \prime} \mathrm{W} ; 21^{\circ} 40^{\prime} 02.98^{\prime \prime} \mathrm{S}\right)$, 06.x.1947, no coll., $20^{\prime \prime}$ (Schubart, 1955).

\section{Leptodesmus defensus Hoffman, 1971}

Leptodesmus defensus Hoffman, 1971: 243, Figs. 11-14 (Male holotype and two female paratypes, MZSP, from Fazenda Aceiro, Jataí, 5143'27.24"W; 1753'19.78"S, Goiás, Brazil).

Historical records: BRAZIL. São Paulo: None.

New records: BRAZIL. São Paulo: Pindamonhangaba (4527'20.47"W; 2255'26.09"S), Colégio Socorro, x.1954, A. Eterovic coll., $10^{\text {T } 19} 1$ juv. (IBSP 135).

\section{Leptodesmus dentellus Schubart, 1946}

Leptodesmus dentellus Schubart, 1946: 173, Fig. 5 (Male holotype, MZSP, from Nova Odessa, 47¹7'39.84"W; $22^{\circ} 47^{\prime} 10.37$ "S, Americana, São Paulo, Brazil); Schubart, 1952: 404, 1955: 516; Hoffman, 1971: 245; Fontanetti, 1991: 542.

Historical records: BRAZIL. São Paulo: Americana, Nova Odessa (47¹7'39.84"W; 2247'10.37"S), 24.v.1945, O. Schubart coll., 107 (Schubart, 1946); Analândia, Morro Grande (47³9'44.98"W; 2207'46.62"S), 25.ix.1947, no

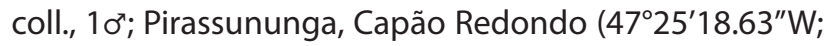
$\left.21^{\circ} 59^{\prime} 58.32^{\prime \prime S}\right)$, 26.xi.1948, no coll., $10^{7} 3$ juv.; Leme

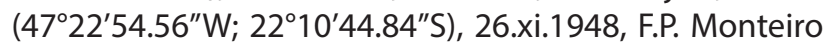

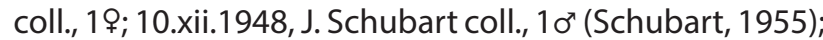
Rio Claro, Horto Florestal (47³3'28.77"W; 22²4'55.87"S), vii.1949, J. Callegari coll., 10'; Campinas, Boa Vista (4703'30.75"W; 2254'35.46"S), v.1949, M. Autuori coll., $20^{\rtimes} 2$ juv. (Schubart, 1955).

New records: BRAZIL. São Paulo: Piracicaba, Campus UNESP (47³7'58.81"W; 2244'11.84"S), 27.v.1940, Piza

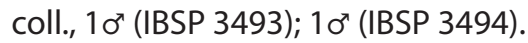

\section{Leptodesmus forceps Brölemann, 1902}

Leptodesmus forceps Brölemann, 1902a: 61, Figs. 30-33 (Syntypes, MZSP, from Itapetininga, 4802'29.39"W; $23^{\circ} 35^{\prime} 12.71^{\prime \prime} \mathrm{S}$, and Santo André, 46² $18^{\prime} 59.66^{\prime \prime} \mathrm{W}_{\text {; }}$ 2346'59.69"S, Alto da Serra (=Serra deParanapiacaba), São Paulo, Brazil); Brölemann, 1909: 68; Schubart, 1955: 527; Hoffman, 1971: 246.

Leptodesmus (Leptodesmus) forceps: Attems, 1938: 16, Fig. 11.

Historical records: BRAZIL. São Paulo: Santo André, Alto da Serra (= Serra de Paranapiacaba) (46 18'59.66"W; $\left.23^{\circ} 46^{\prime} 59.69^{\prime \prime} \mathrm{S}\right)$, no data, nocoll.; Itapetininga (4802'29.39"W; 2335'12.71"S), i.1897, no coll. (Brölemann, 1902a).

\section{Leptodesmus geniculatus Schubart, 1943}

Leptodesmus (Brachyurodesmus) geniculatus Schubart, 1943: 150, Figs. 51-54 (Male holotype, MZSP, from Ilha Seca, Pereira Barreto, 5107'3.91"W; 20³9'17.01"S, São Paulo, Brazil); Schubart, 1955: 516.

Leptodesmus geniculatus: Hoffman, 1971: 246.

Historical records: BRAZIL. São Paulo: Pereira Barreto (5107'3.91"W; 20³9'17.01"S), Ilha Seca, 20.ii.1940, J. Moojen \& H. Berla coll., 30'; Pereira Barreto $\left(51^{\circ} 07^{\prime} 3.91^{\prime \prime} \mathrm{W}\right.$; 20³9'17.01"S), Lussanvira, 16.x.1938, Equipe Instituto Oswaldo Cruz coll., 10'; Itapura $\left(51^{\circ} 27^{\prime} 17.08^{\prime \prime} \mathrm{W}\right.$; $20^{\circ} 37^{\prime} 16.89^{\prime \prime}$ S), 16.x.1938, Equipe Instituto Oswaldo Cruz coll., $10^{\prime \prime}$ (Schubart, 1943); Araçatuba (50²6'00.01"W; $\left.21^{\circ} 12^{\prime} 18.51^{\prime \prime S}\right)$, xii.1926, R. Spitz coll., $10^{\prime \prime}$ (Schubart, 1955).

\section{Leptodesmus godoii Schubart, 1946}

Leptodesmus godoii Schubart, 1946: 169, Fig. 3 (Male holotype, MZSP, from Rio Sapucaí, Corredeira Alegre, São Joaquim da Barra, 4751'35.96"W; 20³5'19.74"S, São Paulo, Brazil); Schubart, 1955: 515, 1956c: 366; Hoffman, 1971: 246, Figs. 15-16.

Historical records: BRAZIL. São Paulo: São Joaquim da Barra (4751'35.96"W; 20³5'19.74"S), 23.vi.1944, M.P. de Godoi coll., 100'169 1 juv. (Schubart, 1946).

\section{Leptodesmus limbatus Schubart, 1955}

Leptodesmus limbatus Schubart, 1955:517, Figs. 7-10 (Male holotype, MZSP, from Novo Horizonte, 49¹3'13.71"W; 21ํำ'12.00"S, São Paulo, Brazil); Hoffman, 1971: 247.

Historical records: BRAZIL. São Paulo: Novo Horizonte

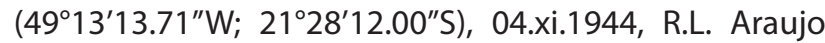
coll., 30' (Schubart, 1955).

\section{Leptodesmus rostratus Schubart, 1955}

Leptodesmus rostratus Schubart, 1955: 518, Figs. 15-16 (Male holotype, MZSP, from Franca, 47²3'24.37"W; 20³2'11.27"S, São Paulo, Brazil); Hoffman, 1971: 247. 
Historical records: BRAZIL. São Paulo: Franca (47²3'24.37"W; 20³2'11.27"S), x.1910, E. Garbe coll., 20" 29 (Schubart, 1955).

\section{Leptodesmus rubicundus Schubart, 1960}

Leptodesmus rubicundus Schubart, 1960b: 456, Figs. 6-7 (Male holotype, MZSP, from Departamento de Botânica, campus da USP, 4643'50.60"W; 2333'41.71"S, São Paulo, São Paulo, Brazil); Hoffman, 1971: 247.

Historical records: BRAZIL. São Paulo: São Paulo, Campos Elíseos (46³8'46.36"W; 2331'57.20"S), 04.xi.1955, M.G. Ferri coll., 19; ii.1956, M.G. Ferri coll., 10"; 29.ix.1956, A.B. Joly coll., 1 ( (Schubart, 1960).

\section{Leptodesmus serrulatus Schubart, 1955}

Leptodesmus serrulatus Schubart, 1955: 518, Figs. 12-14 (Male holotype, MZSP, from Olímpia, 4854'38.76"W; 2044'14.92"S, São Paulo, Brazil); Hoffman, 1971: 248.

Historical records: BRAZIL. São Paulo: Olímpia (4854'38.76"W; 2044'14.92"S), xi.1916, E. Garbe coll., $10^{\prime \prime}$ (Schubart, 1955).

\section{Leptodesmus stimulatus Schubart, 1960}

Leptodesmus stimulatus Schubart, 1960: 459, Fig. 9 (Male holotype, MZSP, from Feiticeiro, Santo Anastácio, 5139'10.43"W; 2158'29.85"S, São Paulo, Brazil); Hoffman, 1971: 248.

Historical records: BRAZIL. São Paulo: Santo Anastácio (51³9'10.43"W; 2158'29.85"S), Feiticeiro, 20.i.1950, O. Schubart coll., 10' 1\%; Santo Anastácio (51³9'10.43"W; $\left.21^{\circ} 58^{\prime} 29.85^{\prime \prime S}\right)$, Palmitalzinho, 19.i.1950, O. Schubart coll., $10^{7}$ (Schubart, 1960).

\section{Leptodesmus tridentatus Schubart, 1960}

Goyazodesmus tridentatus Schubart, 1960: 443, Fig. 6 (Male holotype, MZSP, from Uberlândia, 48 16'32.99"W; 1854'54.08"S, Minas Gerais, Brazil).

Leptodesmus geniculatus: Hoffman, 1971: 248.

Historical records: BRAZIL. São Paulo: None.

New records: BRAZIL. São Paulo: Itirapina, Estação

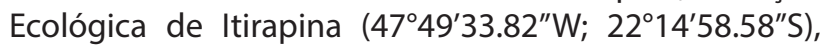
30.iv-05.v.2001, Equipe Biota coll., $10^{\top} 2 \%$ (IBSP 1934).

\section{Leptodesmus vagans Schubart, 1944}

Leptodesmus vagans Schubart, 1944: 356, Figs. 27-29 (Male holotype, MZSP from Fazenda Pedra Branca, Pirassununga, $\quad 47^{\circ} 25^{\prime} 28.16^{\prime \prime} \mathrm{W}$; 2200'19.09"S, São Paulo, Brazil); Schubart, 1955: 516; Hoffman, 1971: 248.
Historical records: BRAZIL. São Paulo: Pirassununga

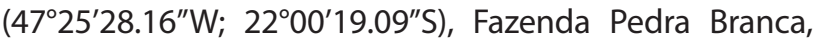
11.i.1942, J. Gaspar \& O. Schubart coll., 7o" 4\%; 15.ii.1942, J. Gaspar \& O. Schubart coll., 20’ 295 juv.; 22.vi.1942, J. Gaspar coll., 1 juv. (Schubart, 1944); Pirassununga (47²5'28.16"W; 22 00'19.09"S), 28.ix.1943, no coll., 3 undetermined (Schubart, 1955); 21-29.v.1940, A. Boggi coll., 1 우 $10^{\prime} 2$ undetermined; 27.vi.1940, A. Boggi coll., 10' 2q; 25.ix.1940, A. Boggi coll., 10'; 18.x.1940, A. Boggi coll., $1 \sigma^{7}$ 1\%; 16.xi.1940, A. Boggi coll., 1o'19;17.iii.1941, no coll., 1 undetermined 1 juv.; 12.v.1941, no coll., 10'19; 08-20.v.1942, no coll., 3 ㅇ (Schubart, 1944); 12-16.vii.1942, no coll., 10' 19; 29.x.1942, no coll., 10'; 28.xi.1942, no coll., 10"; 17.vi.1942, H. Rosa coll., 10'; Mogi Guaçu (4656'35.70"W; $22^{\circ} 22^{\prime} 04.57^{\prime \prime}$ ), 31.vii.1941, no coll., 30' 1 juv.; Porto Ferreira (47²6'55.93"W; $\left.21^{\circ} 50^{\prime} 45.10^{\prime \prime S}\right)$, 17.i.1940, no coll., 1 ㅇ 1 juv.; 21.v.1941, no coll., $20^{\top 1}$ (Schubart, 1944).

\section{Leptodesmus arcanus Schubart, 1944}

Leptodesmus (Leptodesmus) arcanus Schubart, 1944: 348, Figs. 18-19 (Male holotype, MZSP, from Pirassununga, 47²5'28.16"W; 2200'19.09"S, São Paulo, Brazil); 1955: 523.

Historical records: BRAZIL. São Paulo: Pirassununga,

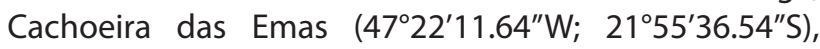
01.xii.1942, L. Gaspar coll., $10^{\prime \prime}$ (Schubart, 1944); Cachoeira das Emas, no data, no coll., $10^{\top}$ (Schubart, 1955).

Remarks: Species under uncertain generic status (Hoffman, 1971).

\section{Leptodesmus bocainae Schubart, 1946}

Leptodesmus bocainae Schubart, 1946: 176, Fig. 7 (Male holotype, MZSP, from Fazenda Nova California, Serra da Bocaina, 44³4'57.77"W; 22³8'45.47"S, São Paulo, Brazil); Schubart, 1955: 527, Fig. 24.

Historical records: BRAZIL. São Paulo: São José dos Barreiros, Serra da Bocaina (44³4'57.77"W; $22^{\circ} 38^{\prime} 45.47^{\prime \prime}$ ), 25.viii.1941, J. d'Amico coll., 40' 5 ㅇ (Schubart, 1946).

Remarks: Species under uncertain generic status (Hoffman, 1971).

\section{Leptodesmus clavaria Brölemann, 1902}

Leptodesmus clavaria Brölemann, 1902b: 687-690, Figs. 33-35, plate 7, 26-29 (Male holotype from Fazenda Nova Niágara [see remarks], 49²8'00.02"W; $23^{\circ} 00^{\prime} 19.02^{\prime \prime}$, municipality not defined by author in 1920, São Paulo, Brazil); 1909: 65; Attems, 1938: 13, Fig. 7; Schubart, 1955: 526.

Historical records: BRAZIL. São Paulo: Batista Botelho (49²8'00.02"W; 2300'19.02"S), Fazenda Nova Niágara, no

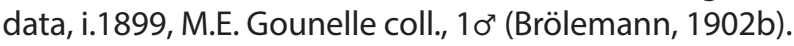


Remarks: Species under uncertain generic status (Hoffman, 1971). The original locality was mentioned erroneously as "Fazenda Nova Nicarágua", and without any reference concerning the municipality. Based in collection records of M.E. Gounelle in the state of São Paulo at the end of the $18^{\text {th }}$ century (see Papavero, 1971: 162-163), the exact location is, indeed, named as "Fazenda Nova Niágara" and located on the municipality of Batista Botelho, outskirts of Cerqueira César.

\section{Leptodesmus corniger Brölemann, 1904}

Leptodesmus (Rachidomorpha) corniger Brölemann, 1904: 87, Figs. 10-14.

Leptodesmus corniger: Brölemann, 1909: 66; Schubart, 1955: 523.

Alocodesmus corniger: Attems, 1931: 57.

Leptodesmus (Desmoleptus) corniger Attems, 1938:35, Fig. 38.

Historical records: BRAZIL. São Paulo: Santo André (46³2'39.72"W; 2340'33.83"S), Rio Grande, 07.i.1902, C.M. Wacket coll. (Brölemann, 1904).

New records: BRAZIL. São Paulo: Embu-Guaçu (4648'55.16"W; 2349'55.88"S), Parque Estadual da Serra do Mar, Cachoeira do Funil, 31.i.2016, A.D. Silva coll., $10^{7}$ (IBSP 4636); 10' (IBSP 4637).

Remarks: Species under uncertain generic status (Hoffman, 1971).

\section{Leptodesmus didymus Schubart, 1946}

Leptodesmus didymus Schubart, 1946: 182, Fig. 11 (Male holotype, MZSP, from Horto Florestal, Serra da Cantareira, Distrito Tremembé, São Paulo, 46³7'33.94"W; 2327'38.68"S, São Paulo, Brazil); Schubart, 1955: 529, Fig. 29.

Historical records: BRAZIL. São Paulo: São Paulo, Serra da Cantareira, Distrito Tremembé (46³7'33.94"W; $23^{\circ} 27^{\prime} 38.68^{\prime \prime S}$ ), ii.1939, no coll., $10^{\prime \prime}$ (Schubart, 1946); Campos do Jordão (45³5'52.39"W; 2244'12.06"S), 14-29. xii.1944, F. Lane coll., 10` 1\%; iii.1945, P. Wygodzinsky coll., $40^{7} 1$ (Schubart, 1955).

Material examined: BRAZIL. São Paulo: Campos do Jordão (45³5'52.39"W; 2244'12.06"S), 04.vi.1994, L. Porto Neto \& A. Eterovic coll., $10^{\text {` }}$ (IBSP 1060).

Remarks: Species under uncertain generic status (Hoffman, 1971).

\section{Leptodesmus furcilla Brölemann, 1902}

Leptodesmus furcilla Brölemann, 1902a: 62, Figs. 34-40 (Two syntypes male and female, MZSP, from Alto da Serra (= Serra de Paranapiacaba), Santo André, 46¹9'01.57"W; 2347'08.23"S, São Paulo, Brazil); Brölemann, 1909: 68; Schubart, 1955: 515.
Leptodesmus (Leptodesmus) furcilla: Attems, 1938: 18, Fig. 15.

Historical records: BRAZIL. São Paulo: Santo André, Alto da Serra (= Serra de Paranapiacaba) (46 $19^{\prime} 01.57^{\prime \prime}$; $23^{\circ} 47^{\prime} 08.23^{\prime \prime S}$ ), no data, no coll. (Brölemann, 1902a).

Remarks: Species under uncertain generic status (Hoffman, 1971).

\section{Leptodesmus gilvomelaena Schubart, 1946}

Leptodesmus gilvomelaena Schubart, 1946: 181, Fig. 10 (Male holotype, MZSP, from Jardim São Bento, São Paulo, 46³8'46.22"W; 2330'12.78"S, São Paulo, Brazil); Schubart, 1955: 528; Trajano et al., 2000: 9.

Historical records: BRAZIL. São Paulo: São Paulo, Jardim São Bento $\left(46^{\circ} 38^{\prime} 46.22^{\prime \prime} \mathrm{W}\right.$; 2330'12.78"S), Chácara Morrinhos, 1939, D.B.J. Pickel coll., 20'1\% (Schubart, 1946).

Remarks: Species under uncertain generic status (Hoffman, 1971).

\section{Leptodesmus infaustus Brölemann, 1902}

Leptodesmus infaustus Brölemann, 1902a: 69 (Male holotype, MZSP, from Perus, São Paulo, 4645'19.24"W; 2324'21.04"S, São Paulo, Brazil); Brölemann, 1909: 69; Attems, 1938: 49; Schubart, 1955: 521.

Historical records: BRAZIL. São Paulo: São Paulo, Perus (4645'19.24"W; 2324'21.04"S), x.1896, no coll., $10^{\prime \prime}$ (Brölemann, 1902a).

Remarks: Species under uncertain generic status (Hoffman, 1971).

\section{Leptodesmus intricatus Schubart, 1955}

Leptodesmus intricatus Schubart, 1955: 528, Fig. 25 (Male holotype, MZSP, from Salesópolis, 4552'11"W; 2337'51"S, São Paulo, Brazil).

Historical records: BRAZIL. São Paulo: Salesópolis,

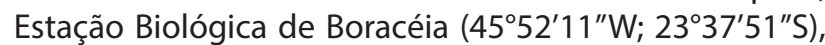
20.xii.1947, P.E. Vanzolini coll., 10¹ㅇ (Schubart, 1955).

Remarks: Species under uncertain generic status (Hoffman, 1971).

\section{Leptodesmus Iaurinhoi Schubart, 1960}

Leptodesmus laurinhoi Schubart, 1960: 460, Figs. 10-12 (Male holotype, MZSP, from Salesópolis, 4552'11"W; 233'51"S, São Paulo, Brazil).

Historical records: BRAZIL. São Paulo: Salesópolis, Estação Biológica de Boracéia (4552'11"W; 2337'51"S), 01.iv.1947, L. Travassos coll., 1\%; Salesópolis, Estação

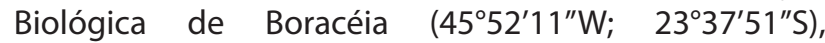


20.xii.1947, P. Vanzolini coll., 29; 05.ii.1959, O. Schubart coll., $10^{\top}$ (Schubart, 1960).

Remarks: Species under uncertain generic status (Hoffman 1971).

\section{Leptodesmus miranda-ribeiroi Schubart, 1955}

Leptodesmus miranda-ribeiroi Schubart, 1955: 524, Fig. 20 (Male holotype, MZSP, from Salesópolis, 4552'11"W; 2337'51"S, São Paulo, Brazil).

Historical records: BRAZIL. São Paulo: Salesópolis, Estação Biológica de Boracéia (4552'11"W; 2337'51"S), 12-20.xii.1947, P.E. Vanzolini coll., $10^{\top} 29$ (Schubart, 1955).

Remarks: Species under uncertain generic status (Hoffman, 1971).

\section{Leptodesmus pardalis Schubart, 1960}

Leptodesmus pardalis Schubart, 1960: 461 (Female holotype, MZSP, from Sítio da Viúva Conde, Itanhaém, 4647'06.02"W; 2410'52.35"S, São Paulo, Brazil).

Historical records: BRAZIL. São Paulo: Itanhaém (4647'06.02"W; 2410'52.35"S), 09.ix.1941, O. Schubart coll., 1 ㅇ (Schubart, 1960).
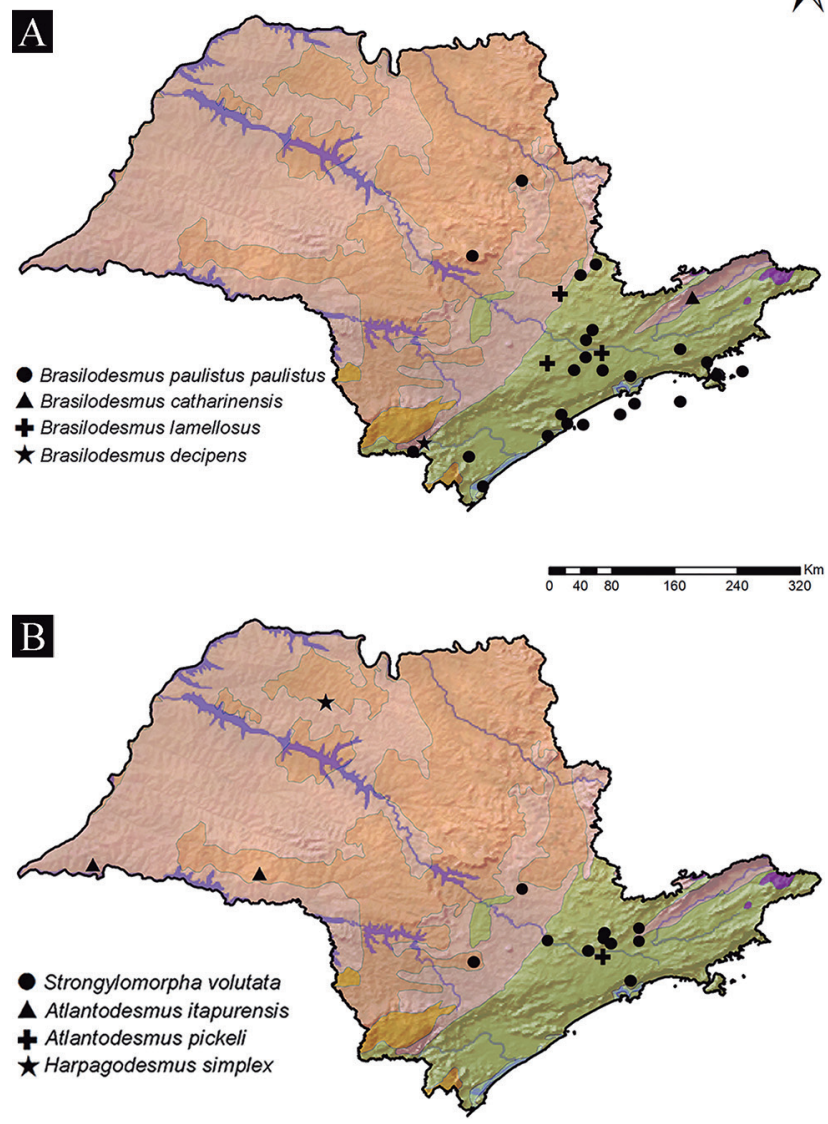

Figure 7. Distribution map of the species belonging to tribes Leptodesmini, Lepturodesmini (A) and Sandalodesmini (B) from São Paulo state, Brazil.
Remarks: Species under uncertain generic status (Hoffman, 1971).

\section{Leptodesmus pubescens Schubart, 1954}

Leptodesmus pubescens Schubart, 1954: 109-112 (Male holotype, MZSP, from Campininha, Piraquara, 4901'44.31"W; 25²7'06.81"S, Paraná, Brazil).

Historical records: BRAZIL. São Paulo: None.

New records: BRAZIL. São Paulo: Apiaí (4851'04.95"W; $\left.24^{\circ} 31^{\prime} 36.00^{\prime \prime S}\right)$, Parque Estadual Turístico do Alto Ribeira (PETAR), Núcleo Caboclo, 21.vii.2016, V.C. Calvanese \& A. Silva coll., $10^{\top}$ (IBSP 4592).

Remarks: Species under uncertain generic status (Hoffman, 1971).

\section{Leptodesmus rubricus Schubart, 1955}

Leptodesmus rubricus Schubart, 1955: 525, Fig. 22 (Male holotype, MZSP, from Campos do Jordão, 4535'51.03"W; 2244'36.61"S, São Paulo, Brazil).

Historical records: BRAZIL. São Paulo: Campos do Jordão (4535'51.03"W; 2244'36.61"S), iii.1945, P. Wygodzinsky

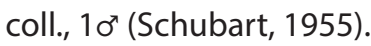

New records: BRAZIL. São Paulo: Salesópolis, Estação Biológica de Boracéia (4552'11"W; 2337'51"S), 08-10. iv.2000, A.D. Brescovit coll., 10"1 1 (IBSP 773).

Remarks: Species under uncertain generic status (Hoffman, 1971).

\section{Leptodesmus silvanus Schubart, 1955}

Leptodesmus silvanus Schubart, 1955: 515, Figs. 3-4 (Male holotype, MZSP, from Alto da Serra (= Serra de Paranapiacaba), Santo André, 46² 18'46.71"W; $23^{\circ} 47^{\prime} 02.73^{\prime \prime S}$, São Paulo, Brazil).

Historical records: BRAZIL. São Paulo: Santo André (4618'46.71"W; 2347'02.73"S), Alto da Serra (= Serra de Paranapiacaba), ix.1908, H. Luederwaldt, $10^{\text {` }}$ (Schubart, 1955).

Material examined: BRAZIL. São Paulo: Santo André, Reserva Biológica do Alto da Serra de Paranapiacaba (4618'46.71"W; 2347'02.73"S), 07-09.x.2007, V. Passanha \& C.V. Janini coll., $10^{\top 1} 1$ juv. (IBSP 3285).

New records: BRAZIL. São Paulo: Salesópolis, Estação

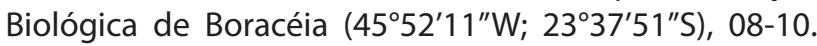
iv.2000, A.D. Brescovit coll., 10' (IBSP 771); v.2001, Equipe Biota coll., $10^{x}$ (IBSP 1897).

Remarks: Species under uncertain generic status (Hoffman, 1971). 


\section{Leptodesmus tucanus Schubart, 1946}

Leptodesmus tucanus Schubart, 1946: 179, Fig. 9 (Male holotype, MZSP, from Fazenda Nova California, Serra da Bocaina, 44³4'57.77"W; 22³8'45.47"S, São Paulo, Brazil); Schubart, 1955: 527.

Historical records: BRAZIL. São Paulo: Fazenda Nova California, Serra da Bocaina (44³4'57.77"W; $\left.22^{\circ} 38^{\prime} 45.47^{\prime \prime S}\right), 25 . v i i i .1941$, J. d'Amico coll., $30^{\prime \prime} 291$ juv. (Schubart, 1946).

Remarks: Species under uncertain generic status (Hoffman, 1971).

\section{Leptodesmus verrucula Brölemann, 1902}

Leptodesmus verrucula Brölemann, 1902a: 83, Figs. 80-81 (Female holotype, MZSP, from Alto da Serra (= Serra de Paranapiacaba), Santo André, 46¹8'39.26"W; 2346'57.17"S, São Paulo, Brazil); Brölemann, 1909: 73; Attems, 1938: 51.

Historical records: BRAZIL. São Paulo: Santo André, Alta da Serra (= Serra de Paranapiacaba) (46²'39.26"W; $\left.23^{\circ} 46^{\prime} 57.17^{\prime \prime S}\right)$, no data, no coll. 1 juv. $q$ (Brölemann, 1902a)

Remarks: Species under uncertain generic status (Hoffman, 1971).

\section{Tribe Lepturodesmini Hoffman, 1975}

(Fig. 7A)

\section{Camptomorpha Silvestri, 1897}

Camptomorpha Silvestri, 1897: 10. Type species: Camptomorpha dorsalis Silvestri, 1897, by subsequent designation of Attems, 1931: 44; 1938: 69 (incorrect description); Hoffman, 1953: 120-124; Schubart, 1955: 510; Jeekel, 1971: 252; Hoffman, 1980: 152.

Phantasmodesmus Verhoeff, 1927: 509. Type species: Leptodesmus pulvillatus Attems, 1898, by monotype; Attems, 1898: 391; 1938: 6 (Syn. with Leptodesmus); Hoffman, 1953: 121 (Syn. with Camptomorpha); Kraus, 1955: 183, 1956: 142.

Eucampesmus Chamberlin, 1941: 489. Type species: Eucampesmus orites Chamberlin, 1941, by original designation; Kraus, 1956: 141 (Syn. with Phantasmodesmus); Hoffman, 1953: 121 (Syn. with Camptomorpha); Jeekel, 1971: 262.

\section{Camptomorpha iheringi (Brölemann, 1902)}

Leptodesmus iheringi Brölemann, 1902a: 84, Figs. 82-89 (Male holotype, MZSP, from Piquete, 4511'10.11"W; 22³6'25.88"S, São Paulo, Brazil); Brölemann, 1909: 69.

Camptomorpha iheringi: Attems, 1938: 71, Fig. 82; Schubart, 1943: 148, 1955: 511.
Historical records: BRAZIL. São Paulo: Piquete

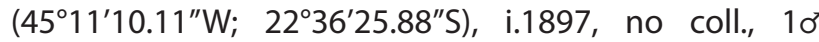
(Brölemann, 1902a).

Remarks: According to Hoffman (1953), C. iheringi is under a generic doubtful position, being the species of Camptomorpha strictu sensu restricted to a small group from northern Andean region and Brazilian Amazonian forest.

\section{Tribe Sandalodesmini Hofmann, 1982} (Fig. 7B)

\section{Sandalodesmus Silvestri, 1902}

Sandalodesmus Silvestri, 1902: 4. Type species: Sandalodesmus bertonii Silvestri, 1902, by monotypy; Brölemann, 1916: 552; Jeekel, 1971: 286; Hoffman, 1980: 155; 1982: 248.

Gonioleptodesmus Schubart, 1958: 29. Type species: Leptodesmus gasparae Schubart, 1944, by original designation. Hoffman, 1967: 34, 1980: 154, 155 (Syn.); Shelley et al., 2000: 103.

\section{Sandalodesmus araujoi Schubart, 1946}

Leptodesmus araujoi Schubart, 1946: 171, Fig. 4 (Male holotype, MZSP, from Rodrigues Alves, São Manuel, 48³4'00.13"W; 2244'12.63"S, São Paulo, Brazil); Schubart, 1955: 516.

Leptodesmus (Gonioleptodesmus) araujoi: Schubart, 1958: 29.

Gonioleptodesmus araujoi: Hoffman, 1967: 34.

Sandalodesmus araujoi: Hoffman, 1982: 251, Fig. 12.

Historical records: BRAZIL. São Paulo: São Manuel (48³4'00.13"W; 2244'12.63"S), 09-11.xii.1943， R.L. Araujo coll., $10^{\top}$ (Schubart, 1946).

\section{Sandalodesmus gasparae (Schubart, 1944)}

Leptodesmus (Leptodesmus) gasparae Schubart, 1944: 351, Figs. 20-23. (Syntype from Estação Experimental de Caça e Pesca, Pirassununga, $47^{\circ} 25^{\prime} 57.35^{\prime \prime} \mathrm{W} ; 22^{\circ} 00^{\prime} 02.28^{\prime \prime}$, São Paulo, Brazil).

Leptodesmus gasparae: Schubart, 1955: 516.

Leptodesmus (Gonioleptodesmus) gasparae: Schubart, 1958: 29

Gonioleptodesmus gasparae: Hoffman, 1967: 35.

Sandalodesmus gasparae: Hoffman, 1982:256; Fontanetti, 1996: 593-595.

Historical records: BRAZIL. São Paulo: Pirassununga

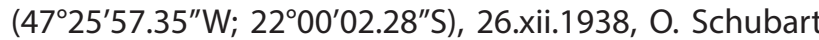
coll., 1 undetermined; 12.v.1941, O. Schubart coll., 2 undetermined; 11.x.1941, J. Gaspar coll., 19; 01.xi.1941, J. Gaspar coll., 10'; 26.x.1942, J. Gaspar coll., 10'; 01-03. xi.1942, J. Gaspar coll., 3o'; 11.i.1942, J. Gaspar coll., 1 juv.; Estação Experimental de Caça e Pesca, 07.x.1940, 
O. Schubart coll., $10^{\prime} 19 ; 25 . x .1940$, O. Schubart coll., $10^{7}$ 1; 27.xi.1940, O. Schubart coll., 10;; 02-16.xi.1940, O. Schubart coll., 760' 269; 24-25.ix.1941 O. Schubart coll., 130"14\%; 01.xi.1941, O. Schubart coll., 10"; 25.ix.1942, O. Schubart coll., 10'19; 12-18.xii.1942, A.L. Gomes \& A.J. Faria coll., 2q; Bonfim, 25.ix.1947, no coll., 640'q; 12.xi.1941, no coll., 10'; Porto Ferreira $\left(47^{\circ} 28^{\prime} 6.30^{\prime \prime} \mathrm{W}\right.$;

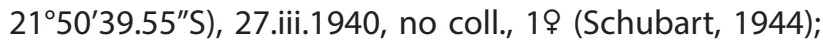
Analândia (47³9'44.40"W; 22 07'42.86"S), Morro Grande, 25.ix.1947, no coll., 30' 1\%; Pitangueiras (48 $13^{\prime} 06.44^{\prime \prime} \mathrm{W}$; $21^{\circ} 00^{\prime} 43.74^{\prime \prime}$ ), Fazenda Três Barras, 24.x.1943, F. Lane

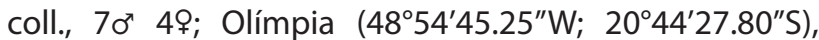
xi.1916, E. Garbe coll., 30'19 (Schubart, 1955).

New records: BRAZIL. São Paulo: Piracicaba (47³8'46.18"W; 2243'50.94"S), Campus ESALQ-USP, 09-10.vii.1994, A. Eterovic coll., $10^{\prime \prime} 19$ (IBSP 1139);

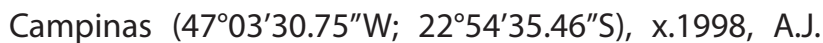
Santos coll., 30' 29 (IBSP 735); Fazenda Santa Genebra $\left(47^{\circ} 38^{\prime} 46.18^{\prime \prime} \mathrm{W} ; 2^{\circ} 43^{\prime} 50.94^{\prime \prime} \mathrm{S}\right), 1993, \mathrm{D}$. Farias \& $\mathrm{A}$. Eterovic coll., $10^{7}$ (IBSP 1079).

\section{Sandalodesmus hippocampus (Schubart, 1944)}

Leptodesmus (Leptodesmus) hippocampus Schubart, 1944: 355, Figs. 24-26 (Syntypes, MZSP, from Usina Corumbataí, 47³7'20.38"W; 22¹3'26.30"S, Rio Claro, São Paulo, Brazil).
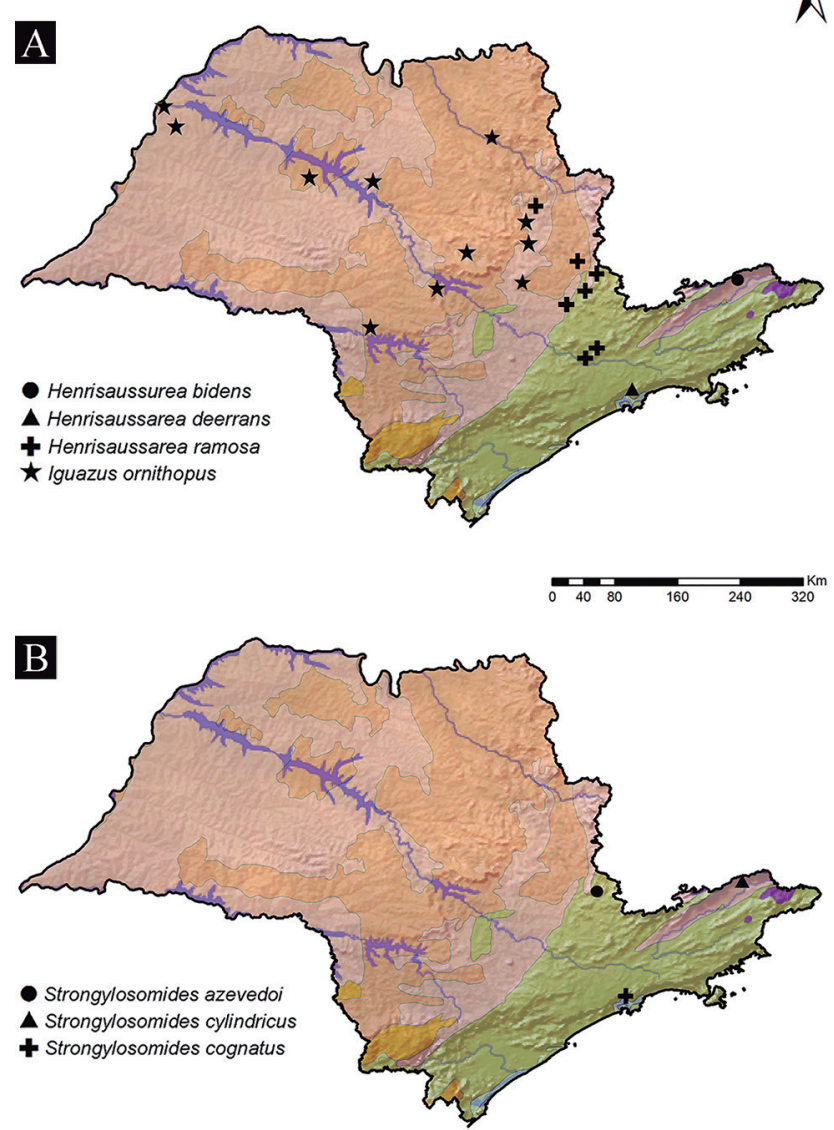

Figure 8. Distribution map of the species belonging to tribe Strongylomorphini (A, B) from São Paulo state, Brazil.
Leptodesmus hippocampus: Schubart, 1955: 516.

Gonioleptodesmus hippocampus: Hoffman, 1967: 35.

Sandalodesmus hippocampus: Hoffman, 1982:256, Fig. 12.

Historical records: BRAZIL. São Paulo: Rio Claro (47³7'20.38"W; 22¹3'26.30"S), 16.xi.1941, J. Gaspar coll., $2 \sigma^{\top}$ (Schubart, 1944).

\section{Sandalodesmus librellus Hoffman, 1982}

Sandalodesmus librellus Hoffman, 1982: 256, Figs. 3-4 (Male holotype, ZSM, from São Paulo, 46³6'42.21"W; $23^{\circ} 34^{\prime} 52.28^{\prime \prime}$, Brazil).

Historical records: BRAZIL. São Paulo: São Paulo

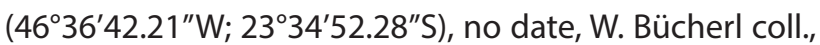
$10^{\prime}$ (Hoffman, 1982).

\section{Tribe Strongylomorphini Hoffman, 1981 (Fig. 8A-B)}

\section{Brasilodesmus Brölemann, 1929}

Brasilodesmus Brölemann, 1929: 35. Type species: Leptodesmus paulistus Brölemann, 1902a, by original designation. Jeekel, 1971: 251; Hoffman, 1971: 260, 1980: 153, 1981: 179, 2009: 561.

\section{Brasilodesmus catharinensis (Brölemann, 1929)}

Leptodesmus (Brasilodesmus) catharinensis Brölemann, 1929: 27, Figs. 38-44 (Male and female syntypes, MNHN, from Jararaca, Santa Catarina, Brazil); Attems, 1938: 27, Fig. 27.

Leptodesmus (Leptodesmus) catharinensis: Attems, 1938: 27. Leptodesmus catharinensis: Schubart, 1953: 106, 1955: 520. Brasilodesmus catharinensis: Hoffman, 1971:262, 1981:180.

Historical records: BRAZIL. São Paulo: São Paulo, Horto Florestal, Distrito Tremembé $\left(46^{\circ} 37^{\prime} 34.13^{\prime \prime}\right.$ W; $23^{\circ} 27^{\prime} 39.15^{\prime \prime}$ S), 08.iv.1944, W. Bücherl coll., 20"3우 (Schubart, 1955).

Remarks: The species may have been introduced into the state by importing plants and seedlings from the Paraná state (Schubart, 1955).

\section{Brasilodesmus lamellosus Brölemann, 1902}

Leptodesmus lamellosus Brölemann, 1902a: 80, Figs. 75-79

(Male holotype, MZSP, from Itatiba, 46 50 '50.29"W; 2300'21.20"S, São Paulo, Brazil); Brölemann, 1909: 70. Leptodesmus (Leptodesmus) lamellosus: Attems, 1938: 24, Fig. 24.

Leptodesmus lamellosus: Schubart, 1955: 526, 1956c: 366. Brasilodesmus lamellosus: Hoffman, 1971: 264, 1981: 180.

Historicalrecords:BRAZIL.SãoPaulo:Itatiba (4650'50.29"W; $23^{\circ} 00^{\prime} 21.20^{\prime \prime S}$ ), vi.1898, no coll., $10^{\top}$ (Brölemann, 1902a); São 


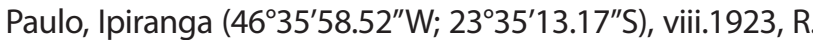
Spix coll., 10'19; vi.1924, R. Spix coll., 3; São Paulo, Freguesia

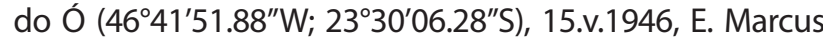

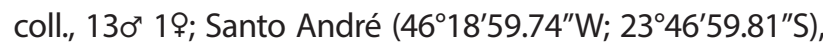
iv.1927, R. Spix coll., 10 (Schubart, 1955).

\section{Brasilodesmus decipens (Brölemann, 1902)}

Leptodesmus decipens Brölemann, 1902a: 77, Figs. 66-69 (Male holotype, MZSP, from Paraná, Brazil); Brölemann, 1909: 66; Schubart, 1954: 108, Fig. 23; Schubart, 1955: 521, Fig. 17.

Leptodesmus (Leptodesmus) decipens: Attems, 1938: 18, Fig. 14.

Brasilodesmus decipens: Hoffman, 1971: 264; 1981: 180.

\section{Historical records: BRAZIL. São Paulo: None.}

Newrecords:BRAZIL. SãoPaulo:Iporanga (48³5'51.20"W;

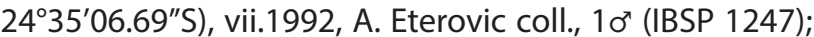
Entrance in cave Morro Grande, 22.iv.2002, D. Candiani \& A.G. Sugmoto coll., $10^{\top}$ (IBSP 1341).

Remarks: The species was previously referenced to São Paulo based on records from Piraquara (Schubart, 1955). However, Piraquara is a municipality of the eastern region of Paraná state, and not to São Paulo.

\section{Brasilodesmus paulistus paulistus (Brölemann, 1902)}

Leptodesmus paulistus Brölemann, 1902a: 59, Figs. 20-29 (Male holotype, MZSP, from Alto da Serra (= Serra de Paranapiacaba), Santo André, 46¹8'35.77"W; 2346'54.82"S, São Paulo, Brazil); Brölemann, 1909: 71.

Leptodesmus (Brasilodesmus) paulistus: Brölemann, 1929: 36.

Leptodesmus (Leptodesmus) paulistus: Schubart, 1945b: 287, 1949: 216.

Leptodesmus paulistus: Schubart, 1955: 519.

Leptodesmus paulistus paulistus: Hoffman, 1971: 265, Figs. 17-18.

Brasilodesmus paulistus paulistus: Hoffman, 1981: 180.

Historical records: BRAZIL. São Paulo: Santo André, Alto da Serra (= Serra de Paranapiacaba) (46 $18^{\prime} 35.77^{\prime \prime} W_{i}$ 2346'54.82"S), no data, no coll., (Brölemann, 1902a); xii.1907, H. Luederwaldt coll., 1\%; 1912, E. Schrebel coll., 10 3\%; 17.vi.1923, R. Spix coll., 20"1\%; Santo André (46³2'38.77"W; 2340'33.85"S), Rio Grande, x.1902, R.V. Ihering coll., 10" (Schubart, 1955); São Sebastião, Ilha

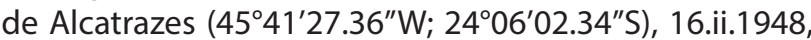

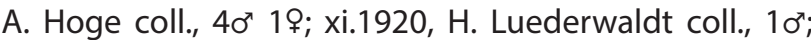
Peruíbe, Ilha da Queimada Grande (46 $40^{\prime} 26.78^{\prime \prime} \mathrm{W}$; $\left.24^{\circ} 29^{\prime} 16.45^{\prime \prime S}\right), 14-22 . i v .1947$, A. Hoge coll., 490 44ㅇ 7 juv. (Schubart, 1949); Ubatuba (4504'27.77"W $23^{\circ} 26^{\prime} 37.28^{\prime \prime}$ S), ilha da Vitória, ix.1907, F. Gunter coll., $10^{\prime \prime}$; São Sebastião $\left(45^{\circ} 24^{\prime} 12.04^{\prime \prime} \mathrm{W} ; 2^{\circ} 48^{\prime} 36.84^{\prime \prime} \mathrm{S}\right)$, 03.i.1949, H. Urban coll., $10^{7} 3$ 9 (Schubart, 1955); Cubatão $\left(46^{\circ} 25^{\prime} 30.19^{\prime \prime} \mathrm{W} ; 23^{\circ} 53^{\prime} 34.82^{\prime \prime} \mathrm{S}\right)$, no data, no coll., undetermined (Brölemann, 1902a); Jacupiranga (4800'27.23"W;
24²'08.23"S), xii.1947, E. Nonato coll., 10"; Peruíbe

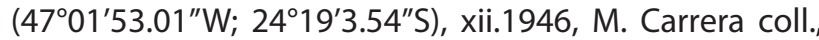
10"; Mogi das Cruzes (46¹1'29.97"W; 2334'30.68"S), ii.1939, W. Bücherl coll., $10^{\top} 1$ juv. (Schubart, 1955); 26.x.1947, M.V. Mendes coll., 10'; Itapecerica da Serra (4651'22.96"W; 2342'58.89"S), João Sarú, 18.x.1944, F. Lane coll., $20^{\top} 1$ 19; São Paulo, Butantã (4643'16.35"W; 2333'52.69"S), 14.iv.1939, P. de Barros coll., 40'3\%; no data, no coll., 20' 6\%; São Paulo, Pinheiros (4641'11.43"W; $\left.23^{\circ} 33^{\prime} 47.02^{\prime \prime S}\right)$, 30.ix.1945, E. Marcus coll., $10^{7}$; Salesópolis, Estação Biológica de Boracéia (4552'11"W; 2337'51"S), 12.xii.1947, P.E. Vanzolini coll., $50^{\circ} 192$ juv. (Schubart, 1955); Amparo (4646'24.91"W; 2242'42.31"S), 08.iii.1943, F. Lane coll., 10'; Amparo, Monte Alegre do Sul (4640'59.52"W; $22^{\circ} 41^{\prime} 26.72^{\prime \prime}$ S), 14.iv.1944, no coll., 1 undetermined (Schubart, 1945b); Brotas (4807'18.25"W; $22^{\circ} 17^{\prime} 0.29^{\prime \prime}$ S), Usina Jacaré, xi.1947, D. Gaspar coll., 20"; 16.ii.1945, J. Schubart coll., 19; ii.1949, D. Gaspar coll., $10^{\circ}$ 1 (Schubart, 1955).

Material examined: BRAZIL. São Paulo: Salesópolis,

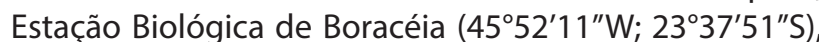
08-10.iv.2000, A.D. Brescovit coll., $10^{\prime \prime}$ (IBSP 769); v.2001, Equipe Biota coll., 10 (IBSP 1884); Peruíbe, Estação Ecológica da Juréia/Itatins (4704'44.02"W; 24¹9'3.54"S), 05.iii.1994, A. Eterovic coll., $10^{\prime 1} 19$ (IBSP 1130); iv.2002, J.P.P. Pena-Barbosa coll., $10^{\pi}$ (IBSP 3743); iv.2002, J.P.P. Pena-Barbosa coll., $10^{\top}$ (IBSP 3742); iii.1997, A.D. Brescovit coll., 10" (IBSP 396); Peruíbe, Ilha da Queimada Grande (4640'26.96"W; $24^{\circ} 29^{\prime} 16.52^{\prime \prime}$ S), iv.1947, Equipe IBSP coll., 20'5우 (IBSP 44); xi.1951, Equipe IBSP coll., 30"7\% (IBSP 73); xi.1951, Equipe IBSP coll., $10^{\prime} 3$ ㅇ 2 juv. (IBSP 72); iv.1947, Equipe IBSP coll., 20'3 (IBSP 211); iv.1947, Equipe IBSP coll., 20 1 우 (IBSP 213); Santo André, Reserva Biológica do Alto da Serra de Paranapiacaba $\left(46^{\circ} 18^{\prime} 37.72^{\prime \prime} W_{i}\right.$ 2346'59.48"S), 14-16.xii.2003, C.A. Rheims e R.P. Indicatti coll., $10^{7}$ (IBSP 1925); São Paulo (46³8'02.24"W; 2333'05.54"S), xii.1990, no coll., 10" (IBSP 514); iv.1939, F. Paes de Barros coll., $10^{\prime 2} 2$ (IBSP 2); iv.1939, F. Paes de Barros coll., 2 ㅇ (IBSP 1); 1943, no coll., 3 9 (IBSP 12); Mogi das Cruzes (46¹1'29.97"W; 2334'30.68"S), ii.1939, W. Bücherl coll., 1 오 (IBSP 35).

New records: BRAZIL. São Paulo: Santa Rita do Passa Quatro (47²8'44.54"W; 2142'25.37"S), 19-24.ii.2002, Equipe Biota coll. 10' (IBSP 2741); Guarujá, Ilha da Moela (461'55.85"W; 2403'00.05"S), 29-31.iii.2009, R.P. Indicatti e G.P. Perroni coll. 20' (IBSP 2868); no data, no coll., $10^{\prime \prime}$ (IBSP 2888); Iguapé (47³3'24.59"W; 2441'57.31"S), Morro do Espia, iv.1994, A. Eterovic coll., 10" (IBSP 1099); Iporanga, Parque Estadual Turístico do Alto Ribeira (PETAR), (48³8'46.90"W; 24²9'13.62"S), Núcleo Santana, 08-14.xii.2002, Equipe Biota coll., $10^{7}$ (IBSP 2786); Iporanga (48³8'46.90"W; 24²9'13.62"S), Parque Estadual Turístico do Alto Ribeira/Parque Estadual Intervales, 1993, R. Pardini \& A. Eterovic coll., $10^{7}$ (IBSP 1068); São Paulo, Parque Estadual Carlos Botelho (4759'43.00"W; $24^{\circ} 03^{\prime} 35.23^{\prime \prime}$ S), 03.ii.2003, D. Pecinini coll., $10^{\prime \prime}$ (IBSP 3266); São Bernardo do Campo, Parque Estoril (46³3'51.82"W;

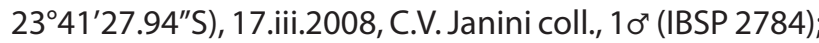


Caraguatatuba $\left(45^{\circ} 25^{\prime} 31.93^{\prime \prime}\right.$ W; $\left.23^{\circ} 37^{\prime} 37.97^{\prime \prime S}\right), x i i .1952$, H. Urban coll., $10^{\top}$ (IBSP 173); Cananéia, Ilha do Cardoso (47 $\left.56^{\prime} 32.99^{\prime \prime} \mathrm{W} ; 5^{\circ} 08^{\prime} 06.82^{\prime \prime} \mathrm{S}\right)$, no data, A. Eterovic coll., $10^{7}$ (IBSP 1067).

\section{Strongylomorpha Silvestri, 1897}

Strongylomorpha Silvestri, 1897: 357. Type species: Strongylomorpha vanvolxemi Silvestri, 1897, by monotypy; Jeekel, 1971: 289; Hoffman, 1980: 155, 1981: 171, 1999.

Erythrodesmus Silvestri, 1902: 4. Type species: Odontopeltis bovei Silvestri, 1895, by monotypy; Brölemann, 1916: 552; Attems, 1938: 198; Jeekel, 1971: 274. Hoffman, 1981: 171 (Syn.).

Neocamptomorpha Schubart, 1953: 96. Type species: Neocamptomorpha araucariae Schubart, 1953, by original designation; Jeekel, 1971: 274. Hoffman, 1981: 171 (Syn.).

Camptomorphoides Schubart, 1962: 251. Type species: Leptodesmus boh/si Attems, 1898, by original designation; Shelley et al., 2000: 89. Hoffman, 1981: 171 (Syn.).

\section{Strongylomorpha volutata (Brölemann, 1902)}

Leptodesmus volutatus Brölemann, 1902a: 75, Figs. 61-65 (Syntypes, MZSP, from Itapetininga, 4809'35.26"W; $23^{\circ} 38^{\prime} 23.67^{\prime \prime} \mathrm{S}$, and Belém, 46³5'28.32"W; $23^{\circ} 32^{\prime} 49.50^{\prime \prime} \mathrm{S}$, and Santo André, Alto da Serra (= Serra de Paranapiacaba), 46² $18^{\prime} 32.54^{\prime \prime W}$; 2346'58.75"S, São Paulo, Brazil); Brölemann, 1909: 73.

Camptomorpha volutatus: Schubart, 1954: 120, 1955: 511. Camptomorphoides volutatus: Schubart, 1962: 252. Strongylomorpha volutata: Hoffman, 1981: 179.

Historical records: BRAZIL. São Paulo: Santo André, Alto da Serra (= Serra de Paranapiacaba) $\left(46^{\circ} 18^{\prime} 32.54^{\prime \prime} W\right.$; $\left.23^{\circ} 46^{\prime} 58.75^{\prime \prime} \mathrm{S}\right)$, no data, no coll.; Itapetininga (4809'35.26"W; 233'23.67"S), i.1897, no coll.; Belém, Juqueri $\left(46^{\circ} 35^{\prime} 28.32^{\prime \prime} \mathrm{W}\right.$; 2332'49.50"S), no data, no coll. (Brölemann, 1902a); São Paulo, Distrito Tremembé (463'34.00"W; 2327'40.54"S), Horto Florestal, ii.1939, no coll., 10"; iv.1939, P. de Barros coll., 10'39; 08.iv.1944, W. Bücherl coll., 2o’ 2o; 04.viii.1944, J. Schubart coll., 10'; 19.xi.1944, E. Nonato coll., 19; Freguesia do Ó (4641'51.51"W; 2330'07.00"S), 15.v.1947, E. Marcus coll., $10^{\top}$ (Schubart, 1955).

New records: BRAZIL. São Paulo: Cotia (4655'09.46"W; $\left.23^{\circ} 36^{\prime} 25.21^{\prime \prime S}\right), x .1983$, R. Lopes coll., $10^{\prime \prime}$ (IBSP 478); Franco da Rocha (4643'52.16"W; 2319'37.20"S), iii.1985,

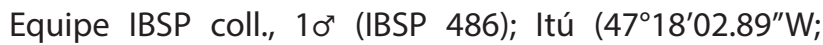
$\left.23^{\circ} 16^{\prime} 19.77^{\prime \prime S}\right)$, iii.1987, R. D'Ávila coll., 20' 1 \% (IBSP 507); Mogi das Cruzes (46¹1'29.70"W; 2334'30.69"S), iv.1939, W. Bücherl coll., 90'7 (IBSP 17); Santa Isabel (46¹3'28.61"W; 2319'04.17"S), 02.i.2003, M. Bedim coll., $10^{7}$ (IBSP 1344); São Paulo, Jardim São Bento, Cháraca Morrinhos (46³8'45.38"W; 2330'13.20"S), 1939, D.B.J. Pickel coll., $10^{\prime \prime}$ (IBSP 33); Piracicaba (47038'53.75"W; $\left.22^{\circ} 44^{\prime} 15.97^{\prime \prime S}\right), x i .1992$, A. Eterovic coll., $10^{7}$ (IBSP 1153).

\section{Tribes incertae sedis \\ (Figs. 9A-B, 10A-B)}

\section{Atlantodesmus Hoffman, 2000}

Atlantodesmus Hoffman, 2000: 101-113. Type species: Leptodesmus (Odontopeltis) eimeri Attems, 1898, by original designation; Bouzan et al. 2017.

lemanja Hoffman, 2000: 101-113. Type species: I. teresa Hoffman, 2000, by original designation and monotypy; Bouzan et al., 2017 (Syn.).

\section{Atlantodesmus itapurensis (Schubart, 1943)}

Leptodesmus (Desmoleptus) itapurensis Schubart, 1943: 148, Figs. 48-50 (Male holotype, MZSP, from Itapura, 5127'14.53"W; 20³7'21.56"S, Andradina, São Paulo, Brazil).

Leptodesmus buecherli: Schubart, 1955: 514, Fig. 1 (Three males and one female syntypes, MZSP, from Distrito de Santana, São Paulo, 46³7'34.79"W; 2329'53.45"S, São Paulo, Brazil); Bouzan et al., 2017 (Syn.).

Atlantodesmus itapurensis: Hoffman, 2000: 106; Bouzan et al., 2017.

Historical records: BRAZIL. São Paulo: Itapura (5127'14.53"W; 20³7'21.56"S), 16.x.1938, Equipe Instituto Oswaldo Cruz coll., 10" (Schubart, 1943); Araçatuba,
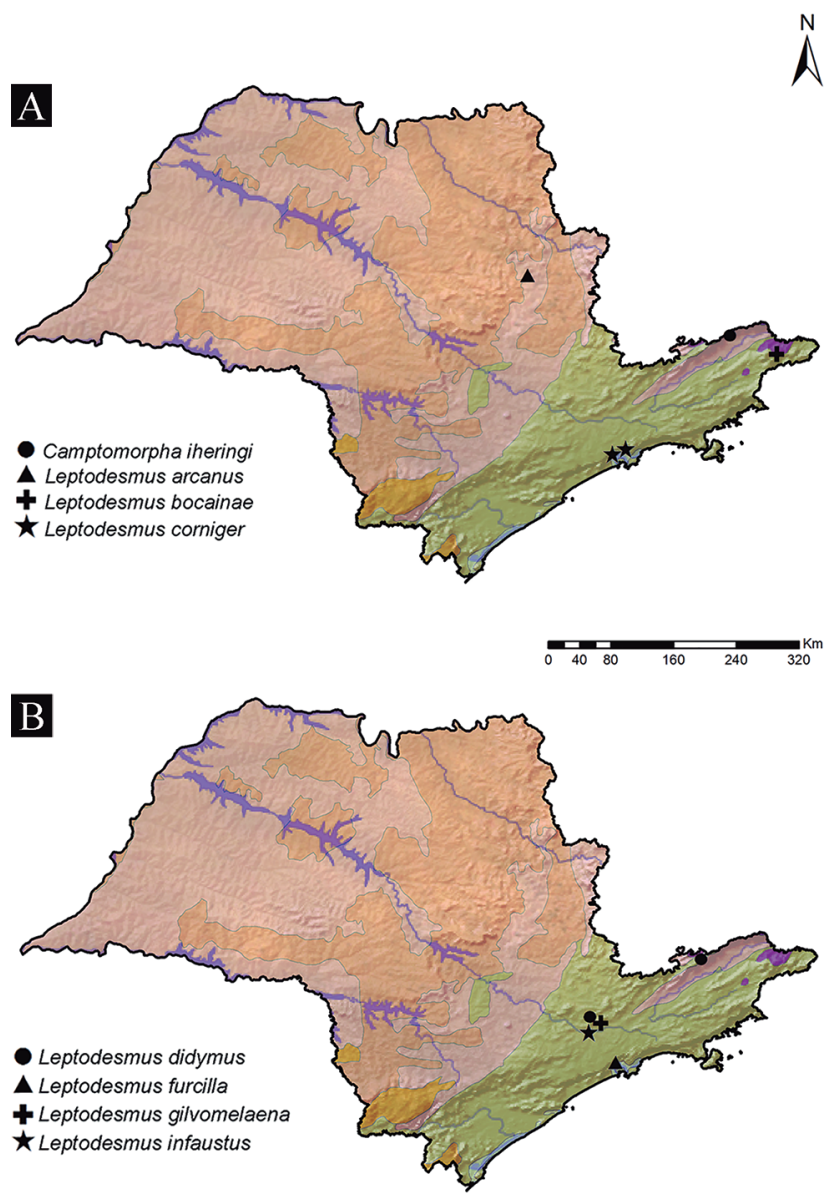

Figure 9. Distribution map of the species belonging to genera with no tribal designation (A, B) from São Paulo state, Brazil. 
Anhangaí $\left(50^{\circ} 26^{\prime} 02.45^{\prime \prime} \mathrm{W} ; 21^{\circ} 12^{\prime} 41.93^{\prime \prime} \mathrm{S}\right)$, xii.1926, R. Spix coll., 10'; São Paulo, Santana (46³7'34.79"W; 2329'53.45"S), 1940, W. Bücherl coll., 30'19 (Schubart, 1955); Monte Aprazível (4942'40.04"W; 2046'06.46"S), 09.i.1950, O.

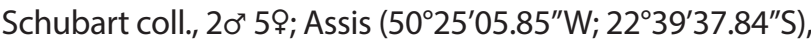
Estação Ecológica de Assis, 25-30.xi.2002, Equipe Biota coll., 140' 10\%; Teodoro Sampaio, Parque Estadual Morro do Diabo (52 $\left.{ }^{\circ} 18^{\prime} 04.17^{\prime \prime} W ; 22^{\circ} 32^{\prime} 07.13^{\prime \prime S}\right), 11-14 . x i .2012$, P.G.B.S. Dias et al. coll., 20 1 \% (Bouzan et al., 2017).

\section{Atlantodesmus pickeli (Schubart, 1946)}

Leptodesmus pickeli Schubart, 1946: 166, Fig. 1 (Male holotype, IBSP, from Jardim São Bento, São Paulo, 46³8'45.47"W; 2330'10.22"S, São Paulo, Brazil); Schubart, 1955: 514.

Atlantodesmus pickeli: Hoffman, 2000: 106; Bouzan et al. 2017.

Historical records: BRAZIL. São Paulo: São Paulo, Jardim São Bento (46³8'45.47"W; 2330'10.22"S), Chácara Morrinhos, 1939, D.B.J. Pickel coll., $10^{\top} 1$ ( (Schubart, 1946).

\section{Harpagodesmus Schubart, 1960}

Harpagodesmus Schubart, 1960: 454. Type species: Harpagodesmus simplex Schubart, 1960, by monotypy. Hoffman, 1980: 154; Shelley et al., 2000: 104.
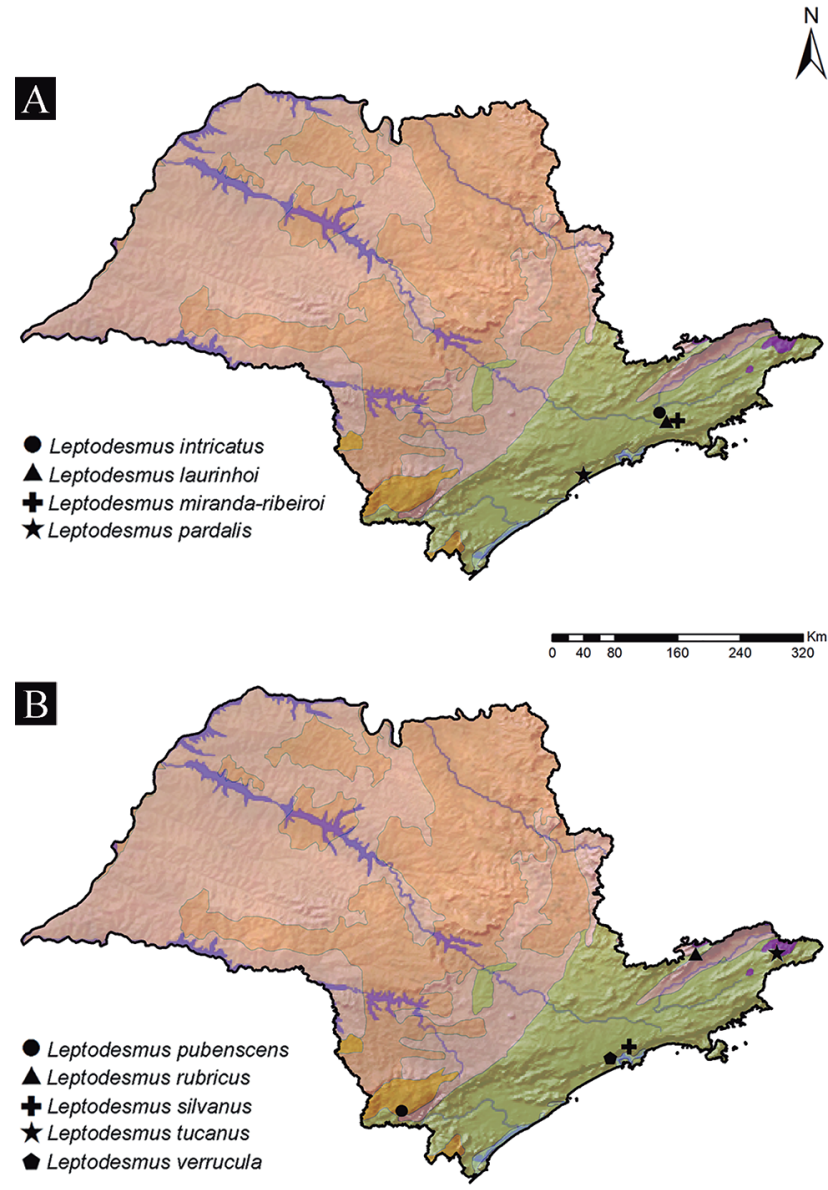

Figure 10. Distribution map of the species belonging to genera with no tribal designation (A, B) from São Paulo state, Brazil.

\section{Harpagodesmus simplex Schubart, 1960}

Harpagodesmus simplex Schubart, 1960: 455, Figs. 3-5 (Male holotype, MZSP, from Fazenda São José do Varjão, Monte Aprazível, 4942'32.79"W; $20^{\circ} 46^{\prime}$ 08.72"S, São Paulo, Brazil).

Historical records: BRAZIL. São Paulo: Monte Aprazível (4942'32.79"W; 2046'08.72"S), Fazenda São José do Varjão, 09.i.1950, O. Schubart coll., 10 1\% 19; 10.i.1950, O. Schubart coll., $10^{\top}$ (Schubart, 1960).

\section{Henrisaussurea Hoffman, 1971}

Henrisaussurea Hoffman, 1971: 267. Type species: Leptodesmus corcovadis Brölemann, 1902, by original designation. Hoffman, 1980: 154; Shelley, 2000: 105; Hoffman, 2009: 561.

\section{Henrisaussurea bidens (Brölemann, 1902)}

Leptodesmus bidens Brölemann, 1902a: 66, Figs. 45-49 (Male holotype, MZSP, from Piquete, 4510'43.13"W; 22³6'48.72"S, São Paulo, Brazil); Brölemann, 1909: 64; Schubart, 1946: 193; Schubart, 1955: 522.

Leptodesmus (Leptodesmus) bidens: Attems, 1938: 17, Fig. 13. Henrisaussurea bidens: Hoffman, 1971: 271.

Historical records: BRAZIL. São Paulo: Piquete

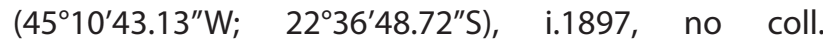
(Brölemann, 1902a).

\section{Henrisaussurea deerrans (Brölemann, 1902)}

Leptodesmus deerans Brölemann, 1902a: 78, Figs. 70-74 (Male holoype from Alto da Serra (= Serra de Paranapiacaba), Santo André, 46¹8'57.09"W; 2347'01.77"S, São Paulo, Brazil); Brölemann, 1909: 67; Schubart, 1946: 194, 1955: 522, Fig. 2.

Leptodesmus (Leptodesmus) deerans: Attems, 1938: 24, Fig. 23.

Henrisaussurea deerrans: Hoffman, 1971: 271.

Historical records: BRAZIL. São Paulo: Santo André, Alto da Serra (= Serra de Paranapiacaba) (46¹8'57.09"W; 234'01.77"S), no data, no coll. (Brölemann, 1902a); 1912, E. Schrebel, $10^{\text {' }}$ (Schubart, 1955).

\section{Henrisaussurea ramosa (Schubart, 1944)}

Leptodesmus (Leptodesmus) ramosus Schubart, 1944; 359, Figs. 30-31 (Male holotype, MZSP, from Fazenda Retiro das Sete Lagoas, Mogi Guaçu, 4656'24.61"W; 22²2'29.29"S, São Paulo, Brazil); Schubart, 1945b: 288. Leptodesmus ramosus: Schubart, 1955: 522, 1956c: 366. Henrisaussurea ramosa: Hoffman, 1971: 272.

Historical records: BRAZIL. São Paulo: Mogi Guaçu, Fazenda Retiro das Sete Lagoas (4656'24.61"W; $\left.22^{\circ} 22^{\prime} 29.29^{\prime \prime S}\right), 31 . v i i i .1941$, O. Schubart coll., $10^{\prime \prime} 4$ ㅇ 
(Schubart, 1944); Monte Alegre do Sul (4640'49.27"W; $22^{\circ} 41^{\prime} 34.67^{\prime \prime}$ ) $)$ x.1942, L. Travassos Filho coll., 30" 19; 27.xi.1942, F. Lane coll., 2o'; 21.vii.1943, no coll., 2o' 29; 30.x-01.xi.1943, no coll., 2o' 2 ' (Schubart, 1945b); Amparo (4646'21.24"W; 2242'31.65"S), 26.x.1948, D. Gaspar coll., 19; Lindóia (46³9'03.75"W; 22³1'27.66"S), 16-17.i.1947, J. Schubart coll., 10' 3\%; Pirassununga, Cachoeira das

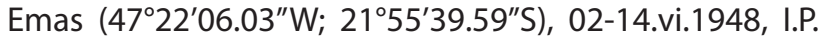
Lima coll., 30" (Schubart, 1955).

Material examined: BRAZIL. São Paulo: Amparo (4646'21.24"W; 2242'31.65"S), x.1974, C. Langen coll., $20^{\top} 4$ 우 (IBSP 200).

New records: BRAZIL. São Paulo: Vinhedo (4659'02.91"W; $23^{\circ} 01^{\prime} 46.51^{\prime \prime}$ ), iv.1983, V. Dragamov coll., 20 1 \% (IBSP 479); Carapicuiba (4649'51.88"W; $23^{\circ} 34^{\prime} 08.44^{\prime \prime}$ S), 03.xi.1998, M. Diez coll., $10^{7}$ (IBSP 692); São Paulo, Parque Estadual do Jaraguá $\left(46^{\circ} 45^{\prime} 20.55^{\prime \prime}\right.$ W; 2327'32.69"S),

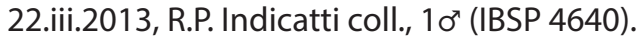

\section{Iguazus Chamberlin, 1952}

Camptomorpha (nec Silvestri, 1897), Attems, 1938: 73 (in part, ornithopus Brölemann); Schubart, 1943: 147 (in part, ornithopus and phoenicopterus). Type species: Leptodesmus ornithopus Brölemann, 1902a.

Iguazus Chamberlin, 1952: 568. Type species: I. leius Chamberlin, 1952, by original designation and monotype; Jeekel, 1971: 267; Hoffman, 1980: 154; Bouzan et al. 2017.

Hoffmanodesmus Schubart, 1962: 255. Type species: Leptodesmus ornithopus (Brölemann 1902a), by original designation. Hoffman, 1965: 219 (Syn.).

\section{Iguazus ornithopus (Brölemann, 1902)}

Leptodesmus ornithopus Brölemann, 1902a:87, Figs. 90-92 (One male and two females syntypes, MZSP, from Cerqueira César, 4640'04.51"W; 2333'34.28"S, São Paulo, Brazil); Brölemann, 1909: 70.

Camptomorpha ornithopus: Attems, 1938: 73; Schubart, 1943: 148, 1952: 403, 1955: 510, 1958: 204; Golovatch et al., 2005: 277.

Camptomorpha phoenicopterus: Schubart, 1943: 147, Figs. 46-47 (Male holotype, MZSP, from Itapura, $51^{\circ} 27^{\prime} 07.06^{\prime \prime} \mathrm{W} ; 20^{\circ} 36^{\prime} 52.48^{\prime \prime}$, São Paulo, Brazil); Schubart, 1962: 255 (Syn.).

Iguazus leius Chamberlin, 1952: 568, Fig. 17 (Male holotype, one male and six female paratypes, $F M N H$, from Cataratas do Iguaçu, Misiones province,

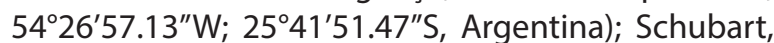
1954: 131; Hoffman, 1965: 221 (Syn.).

Hoffmanodesmus ornithopus: Schubart, 1962: 254.

Iguazus ornithopus: Hoffman, 1965: 221, Fig. 1.

Historical records: BRAZIL. São Paulo: Cerqueira César $\left(46^{\circ} 40^{\prime} 10.78^{\prime \prime} \mathrm{W} ; 23^{\circ} 33^{\prime} 35.09^{\prime \prime} \mathrm{S}\right)$, xii, no coll., $10^{\prime \prime}$ 2 (Brölemann, 1902a); São Manoel (48³4'07.35"W; $\left.22^{\circ} 44^{\prime} 02.82^{\prime \prime S}\right)$, 09.xii.1943, R.L. Araujo coll., $10^{\prime \prime} 1$ \%;
Leme $\left(47^{\circ} 23^{\prime} 05.85^{\prime \prime} \mathrm{W} ; 22^{\circ} 10^{\prime} 53.50^{\prime \prime} S\right), 26 . x i .1948$, F.P. Monteiro \& O. Schubart coll., o" $\$ ; 10 . x i i .1948$, J. Schubart \& F.P. Monteiro coll., $\sigma^{7}$ \%; Pirassununga $\left(47^{\circ} 25^{\prime} 36.23^{\prime \prime} \mathrm{W}\right.$; $21^{\circ} 59^{\prime} 55.81^{\prime \prime}$ ), 26.xi.1948, O. Schubart coll., 10'; Brotas (487'31.35"W; 22¹6'47.57"S), 11.ii.1945, J. Schubart

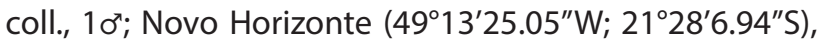
04.xi.1944, R.L. Araujo coll., 40"19; Avanhandava (49 56'46.60"W; $\left.21^{\circ} 27^{\prime} 42.81^{\prime \prime S}\right), 1903$, E. Garbe coll., 30' (Schubart, 1955); Itapura (5129'02.82"W; 20³8'29.93"S), 16.x.1938, Equipe Instituto Oswaldo Cruz coll., 10" (Schubart, 1943).

New records: BRAZIL. São Paulo: Piracicaba (47³9'00.03"W; 2243'59.56"S), 09.xi.1992, A. Eterovic coll., $10^{\top} 3$ (IBSP 1146); Ribeirão Preto, Floresta de Santa Tereza (4750'51.36"W; 2113'09.86"S), 06-11.i.2006, I. Cizauskas coll., 10' 19 (IBSP 3576).

\section{Strongylosomides Attems, 1901}

Strongylosomides Attems, 1901: 89. Type species: Leptodesmus (Strongylosomides) petropolites by monotypy, proposed as a subgenus of Leptodesmus; Attems, 1931: 51 (status changed); 1938: 97; Schubart, 1955: 510; Hoffman, 1979b: 19, 1980: 155.

\section{Strongylosomides azevedoi (Schubart, 1945)}

Leptodesmus azevedoi Schubart, 1945b: 306, Figs. 4-5 (Male holotype, MZSP, from Amparo, 4646'24.32"W; 2242'25.14"S, São Paulo, Brazil); Schubart, 1955: 525. Strongylosomides azevedoi: Hoffman, 1979b: 23.

Historical records: BRAZIL. São Paulo: Amparo (4646'24.32"W; 2242'25.14"S), 21.vii.1943, no coll., 1 undetermined; 30.x-01.xi.1943, no coll., 40 19 2 juv. (Schubart, 1945b).

\section{Strongylosomides cylindricus (Brölemann, 1902)}

Leptodesmus cylindricus Brölemann, 1902a: 71, Figs. 50-55 (Male holotype, MZSP, from Piquete, 4510'44.99"W; 22³6'53.51"S, São Paulo, Brazil); Brölemann, 1909: 66. Strongylosomides cylindricus: Attems, 1938: 98, Fig. 115; Schubart, 1955: 510; Hoffman, 1979a: 23.

Historical records: BRAZIL. São Paulo: Piquete

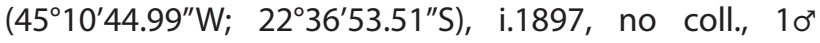
(Brölemann, 1902a).

\section{Strongylosomides cognatus (Brölemann, 1902)}

Leptodesmus cognatus Brölemann, 1902a: 73, Figs. 56-60 (Male holotype, MZSP, from Alto da Serra (= Serra de Paranapiacaba), Santo André, 46²'59.55"W; 2347'00.02"S, São Paulo, Brazil); Brölemann, 1909: 65. Camptomorpha cognatus: Attems, 1938: 72, Fig. 84; Schubart, 1943: 148, 1955: 511.

Camptomorphoides cognatus: Schubart, 1962: 254.

Strongylosomides cognatus: Hoffman, 1979a: 23. 
Historical records: BRAZIL. São Paulo: Santo André, Alto da Serra (= Serra de Paranapiacaba) $\left(46^{\circ} 18^{\prime} 59.55^{\prime \prime}\right.$ W; $23^{\circ} 47^{\prime} 00.02^{\prime \prime} \mathrm{S}$ ), no data, no coll., $10^{\text {* }} 19$ (Brölemann, 1902a).

\section{CONCLUSIONS}

Since the chelodesmidan fauna has been studied in Brazil from the beginning of the $19^{\text {th }}$ century, 11 genera and 68 species were recorded from the state of São Paulo (Schubart, 1955), including erroneously some species recorded from the municipalities of Itatiaia and Rezende from the state of Rio de Janeiro (Schubart, 1955). Based on the data presented herein, 64 species (of ca. 800 species) and 15 genera (of 171 genera) are recorded from São Paulo. Among these species, 30 have only one register documented in the state, and 11 species occurs in other states, as Eurydesmus aguirrei, Leptodesmus godoii, Leptodesmus tridentatus, Brasilodesmus lamellosus, Henrisaussurea ramosa (Minas Gerais state), Leptodesmus defensus (Goiás state), Brasilodesmus decipiens, Leptodesmus pubescens (Paraná state), Brasilodesmus catharinensis (Santa Catarina and Paraná states), Atlantodesmus itapurensi (Mato Grosso Sul state), and Iguazus ornithopus (Mato Grosso do Sul state and Argentina). The most distributed species in São Paulo is Brasilodesmus paulistus paulistus (Strongylomorphini) with 52 records. For 12 species, new records in the state were added, and four species were recorded for the first time to São Paulo (Leptodesmus defensus, L. tridentatus, L. pubescens and Brasilodesmus decipiens).

The occurrence of the genus Camptomorpha (Lepturodesmini) is under doubt, since the species $C$. iheringi is no longer part of the current classification of the genus (Hoffman, 1953), but at the same time that was not included in another genus. The same situation is referred to some species previously described as belonging to Leptodesmus, after the last revision of the genus by Hoffman (1971).

For the state of São Paulo, the main areas with the most number of records are resulted of successive collection efforts by Schubart, especially for regions from Paranapiacaba (known as Alto da Serra) and Pirassununga (and neighboring municipalities). According to Oliveira et al. (2016), most of the Brazilian terrestrial biodiversity (including data of the polydesmidan group) has a pattern of spatially based collection effort, especially in roadside areas. This pattern is highly related to the species of Chelodesmidae from São Paulo, since most of the material was collected in farms and Research Stations (Schubart, 1944, 1955, 1960). It is important to highlight that these regions are also relevant for other millipedes groups in the state, especially for Paradoxosomatidae (Schubart, 1944, 1945a; Nguyen \& Sierwald, 2013), Spirostreptida (Spirostreptidae and Pseudonannolenidae) (Krabbe, 1982; Jeekel, 2004; Iniesta \& Ferreira, 2014), and Spirobolida (genus Rhinocricus) (Schubart, 1951c; Marek et al., 2003).
Since one of the first catalogs of millipedes from Brazil, Schubart has already emphasized the fast rate of environmental degradation from São Paulo state (Schubart, 1955). This fact is more worrying for the species with a single occurrence, since their distribution are from municipalities with small fragments of native forest nowadays. Until the moment, it is clear an underestimation Brazilian millipede fauna. Even for the regions with previous studies, as to southeast region (Brölemann, 1902a; Schubart 1944, 1945b, 1951a, 1955), Pantanal (Golovatch et al., 2005) and central Amazonia (Adis et al., 2002), there are still a large gap on the real richness of the species, making it necessary further studies to provide information concerning the millipede fauna from Brazil.

\section{ACKNOWLEDGMENTS}

This work was supported by CNPq/PIBIC-IC 105077/2015-1 grant to RSB; LFMl was supported partially by a scholarship from CAPES and by grant 2016/24248-0 São Paulo Research Foundation (FAPESP); CNPq 301776/2004-0 grant to ADB and CNPq 143049/2011-9 grant to JPPPB. The authors are grateful to the team from MZSP library for all assistance and to L. Pedroso for correcting the English version.

\section{REFERENCES}

Adis, J.; Foddai D.; Golovatch, S.I.; Hoffman, R.L.; Minelli, A.; de Morais, J.W.; Pereira, L.A.; Scheller, U.; Schileyko, A.A. \& Würmli, M. 2002. Myriapoda at "Reserva Ducke", Central Amazonia/Brazil. Amazoniana, 17(1/2): 15-25.

Attems, C.G. 1898. System der Polydesmiden I. Theil. Denkschriften der Kaiserlichen Akademie der Wissenchaften zu Wien, MathematischNaturwissenschaftliche Klassen, 67: 221-482.

Attems, C.G. 1899. System der Polydesmiden. II. Theil. Denkschriften der Akademie der Wissenschaften Wien, mathematisch-naturwissenschaftliche Klassen, 68: 251-436.

Attems, C.G. 1901. Neue Polydesmiden des Hamburger Museums. Mitteilungen aus dem Naturhistorischen Museum in Hamburg, 18: 83-107.

Attems, C.G. 1931. Die Familie Leptodesmidae und andere Polydesmiden. Zoologica, 30: 1-150.

Attems, C.G. 1938. Polydesmoidea II. Families Leptodesmidae, Platyrhachidae, Oxydesmidae, Gomphodesmidae. Das Tierreich, 69: 1-487.

Bouzan, R.S.; Pena-Barbosa, J.P.P. \& Brescovit, A.D. 2017. Taxonomic review of the genus Atlantodesmus Hoffman, 2000 (Polydesmida: Chelodesmidae). Zootaxa, 4236: 269-290. DOI

Brandt, J.F. 1839. Note relative a la classification des espèces qui composent le genre Polydesmus, et suivie d' une charactéristique de dix espèces nouvelles, ainsi que de quelques remarques sur la distribution géografique des espèces en général. Bulletin Scientifique publié par l'Académie Impériale des Sciences de Saint-Pétersbourg, 5: 307-315.

Brandt, J.F. 1841. Recueil de mémoires relatifs a l'ordre des Insectes Myriapodes, et lus à l'Académie Impériale des Sciences de St.-Pétersbourg. St. Petersbourg, Graeff. 189p.

Brölemann, H.W. 1902a. Myriapodes du Musée de São Paulo. Revista do Museu Paulista, 5: 35-237.

Brölemann, H.W. 1902b. Myriapodes recueillis par M.E. Gounelle au Brésil. Annales de la Société entomologique de France, 71: 649-694. 
Brölemann, H.W. 1904. Myriapodes du Museu Paulista, lle mémorie: Manaos. Revista do Museu Paulista, 6: 63-96.

Brölemann, H.W. 1909. Os myriapodos do Brazil. Catálogos da Fauna Brazileira, 2: 1-94.

Brölemann, H.W. 1916. Essai de classification des Polydesmiens. Annales de la Société entomologique de France, 84: 523-608.

Brölemann, H.W. 1929. Myriapodes recueillis aus Brésil par M. le professeur Caullery, membre de l'institut. Mémoires de la société zoologique de France, 29: 1-37.

Carl, J. 1903. Revision amerikanischer Polydesmiden. Revue suisse de Zoologie, 11:543-562.

Chamberlin, R.V. 1941. On a collection of millipedes and centipedes from Northeastern Peru. Bulletin of the American Museum of natural History, 78(7): 473-535.

Chamberlin, R.V. 1952. Some American polydesmid millipeds in the collection of Chicago Museum of Natural History. Annals of the Entomological Society of America, 45: 553-584.

Cook, 0.F. 1895. Introductory note on the families of Diplopoda. Annals of the New York Academy of Sciences, 9: 1-9.

Fontanetti, C.S. 1991. Morphology of the testicles of some Brazilian species of Diplopoda and their phyllogenetic relations. Revista Brasileira de Zoologia, 7(4): 541-544.

Fontanetti, C.S. 1996. Karyotype of a termitophie species of Diplopoda (Polydesmida, Chelodesmidae). Genetics and Molecular Research, 19(4): 593-595.

Gervais, P. 1847. Myriapodes. In: Walckenaer, 0.A. (Ed.). Histoire naturalle des Insectes Aptères. Paris, Encyclopedique de Roret. p. 1-623.

Gervais, P. 1859. Myriapodes et Scorpions. In: Gervais, P. (Ed.). Animaux nouveaux ou rares recueillis pendantl'expédition dans les parties centrales de L'Amérique du Sud, de Rio de Janeiro à Lima et de Lima au Pará. Paris, P. Bertrand.

Golovatch, S.I.; Hoffman, R.L.; Adis, J.; Marques, M.I.; Raizer, J.; Silva, F.H.O.; Ribeiro, R.A.K.; Silva, J.L. \& Pinheiro, T.G. 2005. Millipedes (Diplopoda) of the Brazilian Pantanal. Amazoniana, 18(3/4): 273-288.

Hoffman, R.L. 1950. The status of the milliped Chelodesmus marxi Cook, and of the family name Chelodesmidae. Proceedings of the Biological Society of Washington, 63: 185-188.

Hoffman, R.L. 1953. The identity of the milliped genus Camptomorpha Silvestri (Polydesmida: Chelodesmidae). Entomological News, 64: 120-124.

Hoffman, R.L. 1954. On three poorly-known neotropical milliped genera (Polydesmida, Eurydesmidae). Proceedings of the Entomological Society of Washington, 56: 215-221

Hoffman, R.L. 1965. Chelodesmid studies I. The status of the generic name Hoffmanodesmus Schubart (Diplopoda: Polydesmida). Papéis Avulsos de Zoologia, 17: 219-223.

Hoffman, R.L. 1967. Chelodesmid Studies III. Notes on the status of Gonioleptodesmus, the description of a new species from Minas Gerais, and a key to the presently known members of the genus (Diplopoda, Polydesmida). Papéis Avulsos do Departamento de Zoologia, 21(4):33-42.

Hoffman, R.L. 1969. Chelodesmid studies. IV. A summary of the tribe Batodesmini, with the description of a new species of Biporodesmus from northwestern Brasil. Papéis Avulsos do Departamento de Zoologia, 22(25): 263-283.

Hoffman, R.L. 1971. Chelodesmid studies V. Some new, redefined, and resurrected Brasilian genera. Arquivos de Zoologia, 20: 225-277.

Hoffman, R.L. 1975. Chelodesmid studies VII. A synopsis of the tribe Lepturodesmini (Polydesmida). Studies on the Neotropical Fauna, 10: 183-200.

Hoffman, R.L. 1976. Chelodesmid studies IX. A synopsis of the new Brazilian tribe Arthrosolaenomeridini (Diplopoda; Polydesmida). Papéis Avulsos de Zoologia, 30: 171-183.
Hoffman, R.L. 1979a. Chelodesmid studies. XII. Two new genera in the brasilian tribe Leptodesmini (Diplopoda, Polydesmida). Papéis avulsos do Departamento de Zoologia, 32: 223-232.

Hoffman, R.L. 1979b. Chelodesmid studies. XV. The identity and status of the generic name Strongylosomides Attems. Myriapodologica, 1: 19-26.

Hoffman, R.L. 1980. Classification of the Diplopoda. Geneva, Muséum D'Histoire Naturelle. 237p.

Hoffman, R.L. 1981. Chelodesmid studies. XIII. A synopsis of the Brasilian tribe Strongylomorphini. Studies on Neotropical Fauna and Environment, 16: 169-184.

Hoffman, R.L. 1982. Chelodesmid studies. XVIII. A synopsis of the genus Sandalodesmus Silvestri, 1902, and proposal of the new tribe Sandalodesmini (Polydesmida: Chelodesmidae). Spixiana, 5(3): 247-259.

Hoffman, R.L. 1990. Chelodesmid studies XX. Millipeds of the new Brasilian tribe Cornalatini (Polydesmida: Chelodesmidae). Papéis Avulsos do Departamento de Zoologia, 37: 23-37.

Hoffman, R.L. 2000. Two new genera of chelodesmid millipeds from southeastern Brazil (Polydesmida: Chelodesmidae). Myriapodologica, 6: 101-113.

Hoffman, R.L. 2009. A new, disjunct, diplopod genus from Espirito Santo, Brasil (Polydesmida: Chelodesmidae). Papéis Avulsos de Zoologia, 49(42): 557-562.

Hoffman, R.L. 2012. New genera for two poorly-known millipeds from Bahia (Polydesmida: Chelodesmidae). Papéis Avulsos de Zoologia, 52(8): 103-110.

Humbert, A. \& Saussure, H. 1872. Études sur les Myriapodes. In: Mission scientifique au Mexique et dans l' Amérique Centrale. Paris, Imprimerie Imperiale. v.6, 2 sec., : 1-211.

Iniesta, L.F.M. \& Ferreira, R.L. 2014. New species of Pseudonannolene Silvestri, 1895 from Brazilian limestone caves with comments on the potential distribution of the genus in South America (Spirostreptida: Pseudonannolenidae). Zootaxa, 3846: 361-397.

Instituto Chico Mendes de Conservação da Biodiversidade (ICMBio). 2016. Sumário Executivo - Livro Vermelho da Fauna Brasileira Ameaçada de Extinção. Brasília, Ministério do Meio Ambiente. 76p.

Instituto Life. 2015. Ecorregiões do Brasil - Prioridades Terrestres e Marinhas, Série Cadernos Técnicos - v.3. Avaiable at: http://institutolife.org/en/ tecnico/publicacoes. Access in: 01/05/2017.

Jeekel, C.A.W. 1971. Nomenclator generum et familiarum Diplopodorum: A list of the genus and family-group names in the Class Diplopoda from the $10^{\text {th }}$ edition of Linnaeus, 1758, to the end of 1957. Monografieen van de Nederlandse Entomologische Vereniging, 5: 1-412.

Jeekel, C.A.W. 2004. A bibliographic catalogue of the "Cambaloidea" (Diplopoda, Spirostreptida). Myriapod Memoranda, 7: 43-109.

Knysak, I. \& Martins, R. 1999. Myriapoda. In: Brandão, C.R.F. \& Cancello, E.M. (Eds.). Biodiversidade do Estado de São Paulo, Brasil. São Paulo, FAPESP. p. 65-72.

Krabbe, E. 1982. Systematik der Spirostreptidae (Diplopoda, Spirostreptomorpha). Abhandlungen und Verhandlungen des Naturwissenschaftlichen Vereins in Hamburg (N.F.), 24: 1-476.

Kraus, 0. 1954. Myriapoden aus Peru, II. Senckenbergiana biologica, 35: 17-55. Kraus, 0. 1955. Myriapoden aus Peru, III. Senckenbergiana biologica, 36: 173-200.

Kraus, 0. 1956. Myriapoden aus Peru, IV. Senckenbergiana biologica, 37: 139-165.

Lindner, V.E.N.; Reip, H.S.; Spelda, J.; Decker, P. \& Voigtländer, K. 2012. Dr. Otto Schubart (17. Februar $1900-08$. November 1962) - Zum 50. Todestag. Schubartiana, 5: 1-40.

Marek, P.E.; Bond, J.E. \& Sierwald, P. 2003. Rhinocricidae Systematics II: A species catalog of the Rhinocricidae (Diplopoda: Spirobolida) with synonymies. Zootaxa, 308: 1-108. 
Mauriès, J.P. 1974. Un cambalide cavernicole du Brésil, Pseudonannolene strinatii n. sp. (Myriapoda-Diplopoda). Revue suisse de Zoologie, 81: 545-549.

Mauriès, J.P. \& Geoffroy, J.J. 2000. Nouvelle description, classification, répartition et variations morphologiques interpopulations $d^{\prime}$ un diplopode troglobie du sud-est du Brésil. Zoosystema, 22: 153-168.

Nguyen, D.A. \& Sierwald, P. 2013. A worldwide catalog of the family Paradoxosomatidae Daday, 1889 (Diplopoda: Polydesmida). Checklist, 9: 1132-1353.

Oliveira, U.; Paglia, A.P.; Brescovit, A.D.; de Carvalho, C.J.B.;Silva, D.P.; Rezende, D.T.; Leite, F.S.F.; Batista, J.A.N.; Barbosa, J.P.P.P.; Stehmann, J.R.; Ascher, J.S.; de Vasconcelos, M.F.; de Marco, P.; Löwenberg-Neto, P.; Dias, P.G.; Ferro, V.G.; Santos, A.J. \& Vanderwal, J. 2016. The strong influence of collection bias on biodiversity knowledge shortfalls of Brazilian terrestrial biodiversity. Diversity and Distributions, 22: 1232-1244.

Papavero, N. 1971. Essays on the history of Neotropical Dipterology, with special reference to collectors (1750-1905). São Paulo, Museu de Zoologia, Universidade de São Paulo. $2 v$.

Pena-Barbosa, J.P.P.; Sierwald, P. \& Brescovit, A.D. 2013. On the largest chelodesmid millipedes: taxonomic review and cladistic analysis of the genus Odontopeltis Pocock, 1894 (Diplopoda; Polydesmida; (helodesmidae). Zoological Journal of the Linnean Society, 169: 737-764.

Peters, W.C. 1865. Übersicht der im Königl. zoologischen Museum befindlichen Myriopoden aus der Familie der Polydesmi, so wie Beschreibungen einer neuen Gattung, Trachyjulus, der Juli und neuer Arten der Gattung Siphonophora. Monatsberichte der Königlich Preußischen Akademie der Wissenschaften zu Berlin, 1864: 529-551.

Pinto-da-Rocha, R. 1995. Sinopse da fauna cavernícola do Brasil (1907-1994). Papéis Avulsos de Zoologia, 39: 61-173.

Pocock, R.I. 1910. Chilopoda and Diplopoda. In: Godman, F. D \& Salvin, 0. (Eds.). Biologia Centrali-Americana, Contributions to the knowledge of the fauna and flora of Mexico and Central America. London, R.H. Porter. p. 1-217.

Saussure, H. 1859. Note sur la famille des Polydesmides, principalment au point de vue des espèces américaines. Linnaea entomológica, 13: 318-327.

Saussure, H. 1860. Essai d'une faune des Myriapodes du Mexique avec la description de quelques espèces des autres parties de l'Amérique. Mémoires de la Société de Physiques et d'Histoire naturelle de Genève, 15: 1-135.

Schubart, 0. 1943. Espécies novas das famílias Strongylosomidae e Leptodesmidae da ordem Proterospermophora do interior dos Estados de São Paulo e Mato Grosso. Papéis Avulsos de Zoologia, 3: 127-164.

Schubart, 0. 1944. Os Diplopodos de Pirassununga. Acta Zoologica Lilloana, 2: $321-440$

Schubart, 0. 1945a. Os Proterospermophora do Distrito Federal (Myriapoda, Diplopoda). Arquivos do Museu Nacional, 38: 1-156.

Schubart, 0. 1945b. Diplópodos de Monte Alegre (Municipio de Amparo, Est. de São Paulo). Papéis Avulsos do Departamento de Zoologia, 6: 283-320.

Schubart, 0. 1946. Contribuição ao conhecimento do gênero Leptodesmus (Fam. Leptodesmidae, Diplopoda). Anais da Academia Brasileira de Ciências, 18: 165-202.

Schubart, 0.1949. 0s Diplopoda de algumas ilhas do litoral Paulista. Memórias do Instituto Butantan, 21: 203-254.

Schubart, 0. 1951a. Sôbre alguns Leptodesmidae do Distrito federal do Brasil e do Estado do Rio de Janeiro. Boletim do Museu Nacional do Rio de Janeiro, 101: 1-15.
Schubart, 0. 1951b. Um novo Eurydesmus de Monte Alegre do Sul (Leptodesmidae, Diplopoda). Revista Brasileira de Biologia, 11: 91-94.

Schubart, 0. 1951c. Contribuição para a fauna do Estado de Sao Paulo II. Os Rhinocricidae (Opisthospermophora, Diplopoda). Anais da Academia Brasileira de Ciências, 23(2): 221-275.

Schubart, 0. 1952. "Leptodesmidae" Brasileiras. I: Três gêneros novos, Goyazodesmus, Rupidesmus e Thaumatodesmus. Revista Brasileira de Biologia, 12: 447-454.

Schubart, 0. 1953 (1954). Sobre os Diplopoda dos estados do Paraná e Santa Catarina. I. Proterospermophora. Arquivos do Museu Paranaense, 10: 77-132.

Schubart, 0. 1955. Materiais para uma fauna do Estado de São Paulo - os Leptodesmidae. Arquivos do Museu Nacional, 42: 507-540.

Schubart, 0. 1956a. "Leptodesmidae" Brasileiras. II: 0 gênero Obricodesmus (Diplopoda, Proterospermophora). Revista Brasileira de Biologia, 16: 341-348

Schubart, 0. 1956b. "Leptodesmidae" Brasileiras. V: Um novo Eurydesmus do litoral do Estado do Rio de Janeiro (Diplopoda, Proterospermophora). Revista Brasileira de Biologia, 16: 453-459.

Schubart, 0. 1956c. "Leptodesmidae" Brasileiras. III: Espécies de Minas Gerais (Diplopoda, Proterospermophora). Revista Brasileira de Biologia, 16: 355-367.

Schubart, 0. 1958. "Leptodesmidae" Brasileiras. VI: Espécies novas do Estado do Rio Grande do Sul (Diplopoda, Proterospermophora). Revista Brasileira de Biologia, 18(1): 23-32.

Schubart, 0. 1960. Leptodesmidae Brasileiras. VIII. Novas espécies do Estado de São Paulo (Diplopoda, Proterospermophora). Revista Brasileira de Biologia, 20: 453-464.

Schubart, 0. 1962. Leptodesmidae Brasileiras. IX: Sobre algumas espécies do gênero Camptomorpha (Proterospermophora, Diplopoda). Revista Brasileira de Biologia, 22: 251-261.

Shear, W. 2011. Class Diplopoda de Blainville in Gervais, 1844. Zootaxa, 3: 159-164. (Zhang, Z.-Q. (Ed.) Animal biodiversity: An outline of higher-level classification and survey of taxonomic richness)

Shelley, R.M.; Sierwald, P.; Kiser, S.B. \& Golovatch, S.I. 2000. Nomenclator generum et familiarum Diplopodorum II. Sofia, Pensoft. 167p. (Pensoft Series Faunistica, 20).

Silhavý, V. 1974. A new subfamily of Gonyleptidae from Brazilian caves, Pachylospeleinae subfam. n. (Opiliones, Gonyleptomorphi). Revue Suisse Zoologie, 81: 893-898.

Silvestri, F. 1895. Viaggio del dottor Alfredo Borelli nella Repubblica Argentina e nel Paraguay. XIV. Chilopodi e Diplopodi. Bollettino del musei di Zoologia e di Anatomia Comparata della Reale Università di Torino, 10: 1-12.

Silvestri, F. 1897. Viaggio del Dott. Alfredo Borelli nel Chaco boliviano e nella Repubblica Argentina. IV. Chilopodi e Diplopod. Bollettino del Musei di Zoologia e di Anatomia Comparata della Reale Università di Torino, 12: $1-11$.

Silvestri, F. 1902. Viaggio del Dr. A. Borelli nel Matto Grosso. VII. Diplopodi. Bollettino del musei di Zoologia e di Anatomia Comparata della Reale Università di Torino, 17: 1-25.

Trajano, E.; Golovatch, S.; Geoffroy, J.J.; Pinto-da-Rocha, R. \& Fontanetti, C.S 2000. Synopsis of Brazilian cave-dwelling millipedes (Diplopoda). Papéis Avulsos do Departamento de Zoologia, 41: 259-287.

Verhoeff, K.W. 1927. Klasse Diplopoda 4. In: Bronn, H.G. (Ed.). Klassen und Ordnungen des Tierreichs.5. Gliederfüssler. Arthropoda, 2. Myriapoda. Leipzig, Akademische Verlagsgesellschaft. p. 481-640. 